\title{
Efficient Access to Cyclic Ureas Via Pd-Catalyzed Cyclization
}

\author{
Mark McLaughlin,* Michael Palucki and Ian W. Davies
}

Department of Process Research, Merck Research Laboratories, Merck \& Co., Inc. Rahway, NJ 07065

mark_mclaughlin@merck.com

Supporting Information

Table of Contents:

General considerations

General procedure A: Reductive alkylation/CSI urea formation

Analytical data for primary ureas

General procedure B: Pd-Catalyzed urea cyclization with dppb

General procedure C: Pd-Catalyzed urea cyclization with X-Phos

Analytical data for prepared cyclic ureas

NMR spectra of primary ureas

NMR spectra of cyclic ureas

30-40 
General considerations: Screening reactions were conducted under a nitrogen atmosphere in $8 \mathrm{~mL}$ vials equipped with magnetic stir-bars and septa. Larger scale preparative reactions were conducted in standard RB-flasks under a nitrogen atmosphere. HPLC grade solvents were used with no additional purification/drying. NMR spectra were recorded using a Bruker DPX-400 spectrometer; ${ }^{1} \mathrm{H}$ NMR recorded at $400 \mathrm{MHz}$ and ${ }^{13} \mathrm{C}$ recorded at $100 \mathrm{MHz}$. TLC was performed using $2.5 \mathrm{~cm} \times 7.5 \mathrm{~cm}$ EMD glass-backed plates (silica gel $60, \mathrm{~F}_{254}, 250 \mu \mathrm{m}$ thickness). Flash column chromatography was performed using EMD silica gel 60, 230-400 mesh. HPLC was performed on an Agilent 1100 Series machine using an Agilent Zorbax (Eclipse XDB C8) column (4.6 mm $\times 150$ $\mathrm{mm}$ ), $0.65 \mathrm{~mL} / \mathrm{min}$ flowrate, $205 \mathrm{~nm}$ detection, $30{ }^{\circ} \mathrm{C}$ column temperature; mobile phase $\mathrm{MeCN} / 0.1 \%$ aq. $\mathrm{H}_{3} \mathrm{PO}_{4} 10 / 90$ for $3 \mathrm{~min}$ then ramp to $60 / 40$ over $10 \mathrm{~min}$ then ramp to $95 / 5$ over 2 min then $95 / 5$ for a further 4 min.

\section{General procedure A}

Reductive alkylation step: To a RB-flask equipped with magnetic stir-bar and temperature probe was charged the arylamine (100 mol\%) and the carbonyl compound (110 mol\%) followed by $i$-PrOAc $(12 \mathrm{~mL} / \mathrm{g}$ of arylamine) and trifluoroacetic acid (200 mol\%). Sodium triacetoxyborohydride (120 mol\%) was added as a solid over $1 \mathrm{~min}$, normally accompanied by an increase in temperature to $\sim 40{ }^{\circ} \mathrm{C}$. After 10 min agitation, the mixture was usually homogeneous and LC analysis indicated complete consumption of the arylamine. A solution of $10 \mathrm{wt} \%$ aqueous $\mathrm{NaOH}$ was added to the mixture until $\mathrm{pH}$ 8-9. The phases were allowed to separate and the organic phase washed with brine then dried over $\mathrm{Na}_{2} \mathrm{SO}_{4}$, filtered and concentrated to afford the crude product. ${ }^{1}$

Urea formation step: To a RB-flask equipped with a magnetic stir-bar and temperature probe was charged with THF ( $<50 \mathrm{ppm} \mathrm{H}_{2} \mathrm{O}, 8 \mathrm{~mL} / \mathrm{mL}$ of CSI) then chlorosulfonyl isocyanate (CSI) (120 mol\%) was added at room temperature. The mixture was cooled to

\footnotetext{
${ }^{1}$ Analytical data for purified reductively alkylated products can be found in the preceding paper. For the present work, the crude products were used directly in the subsequent urea formation.
} 
$-10{ }^{\circ} \mathrm{C}$. A solution of reductively alkylated amine (100 mol\%) in THF : $i-\operatorname{PrOAc}(1: 1)$ ( $<500 \mathrm{ppm} \mathrm{H}_{2} \mathrm{O}, 5 \mathrm{~mL} / \mathrm{g}$ of amine) and added over a 20 min period. An exotherm was observed during this addition (max. temp. $2{ }^{\circ} \mathrm{C}$ ). After $10 \mathrm{~min}$ and with the batch temperature returned to $-10{ }^{\circ} \mathrm{C}$, water $(0.5 \mathrm{~mL} / \mathrm{g}$ of substrate) was added dropwise over a 10 min period. A second exotherm was observed during the water addition (max. temp. $17^{\circ} \mathrm{C}$ ). The mixture was allowed to warm to $\mathrm{rt}$ and aged for $30 \mathrm{~min}$. The mixture was treated with $10 \%$ aq. $\mathrm{NaOH}$ until $\mathrm{pH} 8-9$ and the separated organic phase was washed with brine. The work-up was conducted at $50{ }^{\circ} \mathrm{C}$ to maintain solubility of the product. Azeotropic drying with $i$-PrOAc was conducted at atmospheric pressure under constant volume conditions until the water content was $<250 \mathrm{ppm}$ by KF titration (THF was also removed during this process). The urea was isolated by crystallization.

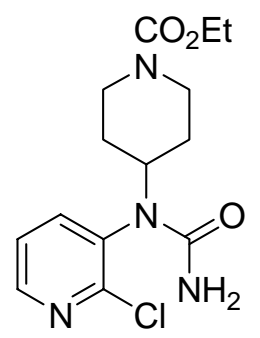

$3 a$

\section{4-[1-(2-Chloro-pyridin-3-yl)ureido]piperidine-1-carboxylic acid ethyl ester (3a):}

Following general procedure A, 3a was obtained as an off-white solid (85\%): ${ }^{1} \mathrm{H}$ NMR (400 MHz, DMSO) $\delta$ 0.73-1.41 (br m, 2H), 1.10 (t, J = 7.2 Hz, 3H), 1.67-2.00 (br m, $2 \mathrm{H}$ ), 2.65-2.93 (br m, 2H), 3.84-4.05 (m, 4H), 4.23-4.36 (m, 1H), 5.84 (s, 2H), 7.45 (dd, $J$ $=7.6,4.8 \mathrm{~Hz}, 1 \mathrm{H}), 7.76(\mathrm{dd}, J=7.6,1.6 \mathrm{~Hz}, 1 \mathrm{H}), 8.37(\mathrm{dd}, J=4.8,1.6 \mathrm{~Hz}, 1 \mathrm{H}) ;{ }^{13} \mathrm{C}$ $\left\{{ }^{1} \mathrm{H}\right\}$ NMR (100 MHz, DMSO) $\delta 14.8,30.0$ and 31.7 (br, rotamers), 43.2, 54.2, 61.0, 124.3, 133.9, 142.2, 149.0, 152.3, 154.7, 156.5. HRMS calcd. for $\mathrm{C}_{14} \mathrm{H}_{19} \mathrm{ClN}_{4} \mathrm{O}_{3}[\mathrm{M}+\mathrm{H}]^{+}$ 327.12184 , found 327.12253 . 


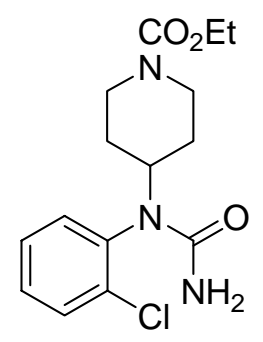

$3 b$

4-[1-(2-Chloro-phenyl)ureido]piperidine-1-carboxylic acid ethyl ester (3b):

Following general procedure A, 3b was obtained as an off-white solid (82\%): ${ }^{1} \mathrm{H}$ NMR (400 MHz, DMSO) $\delta$ 0.74-0.96 (br m, 1H), 1.06 (t, $J=7.2 \mathrm{~Hz}, 3 \mathrm{H}$ ), 1.16-1.35 (br m, 1H), 1.62-1.97 (br m, 2H), 2.66-2.91 (br m, 2H), 3.82-4.04 (m, 4H), 4.21-4.36 (m, 1H), 5.50 (br s, 2H), 7.22-7.27 (m, 1H), 7.31-7.37 (m, 2H), 7.49-7.55 (m, 1H); ${ }^{13} \mathrm{C}\left\{{ }^{1} \mathrm{H}\right\} \mathrm{NMR}$ (100 MHz, DMSO) $\delta$ 15.0, 30.0 and 31.9 (br, rotamers), 43.4, 54.1, 61.1, 128.6, 130.1, 130.9, 133.3, 135.1, 136.9, 154.8, 156.9. HRMS calcd. for $\mathrm{C}_{15} \mathrm{H}_{20} \mathrm{ClN}_{3} \mathrm{O}_{3}[\mathrm{M}+\mathrm{H}]^{+}$ 326.12660 , found 326.12676 .

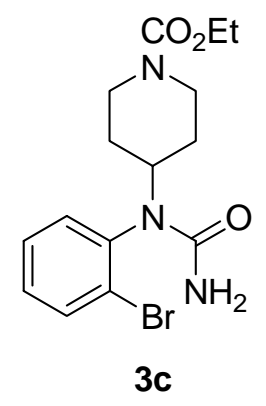

4-[1-(2-Bromo-phenyl)ureido]piperidine-1-carboxylic acid ethyl ester (3c): Following general procedure A, 3c was obtained as an off-white solid (84\%): ${ }^{1} \mathrm{H}$ NMR (400 MHz, DMSO) $\delta$ 0.75-0.95 (m, 1H), 1.67 (t, $J=7.2 \mathrm{~Hz}, 3 \mathrm{H}), 1.21-1.38(\mathrm{~m}, 1 \mathrm{H})$, $1.68-1.80$ (m, 1H), 1.88-1.98 (m, 1H), 2.60-2.91 (br m, 2H), 3.84-4.04 (m, 4H), 4.18-4.29 (m, 1H), 5.46 (br s, 2H), 7.23-7.30 (m, 2H), 7.39 (dt, $J=8.4,2.0 \mathrm{~Hz}, 1 \mathrm{H}), 7.69$ (dd, $J=$ 8.4, 2.0 Hz, 1H); ${ }^{13} \mathrm{C}\left\{{ }^{1} \mathrm{H}\right\}$ NMR (100 MHz, DMSO) $\delta$ 15.0, 31.1 (br d), 43.4, 54.3, 61.1, 126.6, 129.3, 130.3, 133.2, 134.1, 138.6, 154.8, 156.7. HRMS calcd. for $\mathrm{C}_{15} \mathrm{H}_{20} \mathrm{BrN}_{3} \mathrm{O}_{3}$ $[\mathrm{M}+\mathrm{H}]^{+} 370.07608$, found 370.07653 . 


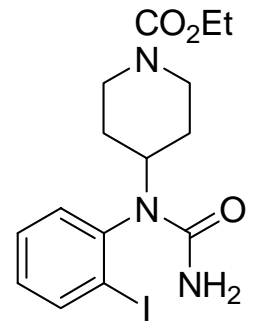

3d

4-[1-(2-Iodo-phenyl)ureido]piperidine-1-carboxylic acid ethyl ester (3d): Following general procedure A, 3d was obtained as an off-white solid (81\%): ${ }^{1} \mathrm{H}$ NMR (400 MHz, DMSO) $\delta$ 0.74-0.88 (m, 1H), 1.03-1.15 (m, 3H), 1.28-1.42 (m, 1H), 1.72-1.81 (m, 1H), 1.94-2.03 (m, 1H), 2.61-2.90 (br m, 2H), 3.80-4.02 (m, 4H), 4.14-4.26 (m, 1H), 5.39 (br s, 2H), $7.07(\mathrm{td}, J=8.0,1.2 \mathrm{~Hz}, 1 \mathrm{H}), 7.23(\mathrm{dd}, J=8.0,1.2 \mathrm{~Hz}, 1 \mathrm{H}), 7.40(\mathrm{td}, J=8.0,1.2$ $\mathrm{Hz}, 1 \mathrm{H}), 7.91(\mathrm{dd}, J=8.0,1.2 \mathrm{~Hz}, 1 \mathrm{H}) ;{ }^{13} \mathrm{C}\left\{{ }^{1} \mathrm{H}\right\}$ NMR (100 MHz, DMSO) $\delta 15.0,30.2$ and 32.1 (rotamers), 43.4 and 43.5 (rotamers), 54.4, 61.1, 105.4, 130.0, 130.2, 132.0, 140.4, 142.3, 154.9, 156.5. HRMS calcd. for $\mathrm{C}_{15} \mathrm{H}_{20} \mathrm{IN}_{3} \mathrm{O}_{3}[\mathrm{M}+\mathrm{H}]^{+}$418.06221, found 418.06301 .

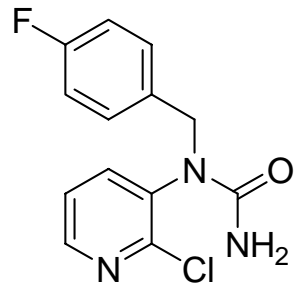

$3 e$

1-(2-Chloro-pyridin-3-yl)-1-(4-fluoro-benzyl)urea (3e): Following general procedure A, 3e was obtained as an off-white solid (88\%): ${ }^{1} \mathrm{H}$ NMR (400 MHz, DMSO) $\delta 4.71$ (br, 2H), $6.05(\mathrm{~s}, 2 \mathrm{H}), 7.08(\mathrm{t}, J=8.8 \mathrm{~Hz}, 2 \mathrm{H}), 7.24(\mathrm{td}, J=8.0,6.0 \mathrm{~Hz}, 2 \mathrm{H}), 7.33$ (dd, $J=$ 8.0, $4.4 \mathrm{~Hz}, 1 \mathrm{H}), 7.45(\mathrm{dd}, J=8.0,2.0 \mathrm{~Hz}, 1 \mathrm{H}), 8.29(\mathrm{dd}, J=4.4,2.0 \mathrm{~Hz}, 1 \mathrm{H}) ;{ }^{13} \mathrm{C}\left\{{ }^{1} \mathrm{H}\right\}$ NMR (100 MHz, DMSO) $\delta 50.8,115.4\left(\mathrm{~d}, J_{\mathrm{CF}}=20.1 \mathrm{~Hz}\right), 124.3,130.8\left(\mathrm{~d}, J_{\mathrm{CF}}=10.1\right.$ $\mathrm{Hz}), 134.5\left(\mathrm{~d}, J_{\mathrm{CF}}=10.1 \mathrm{~Hz}\right), 136.2,141.0,148.7,150.6,157.2,161.8\left(\mathrm{~d}, J_{\mathrm{CF}}=241.0\right.$ $\mathrm{Hz}$ ). HRMS calcd. for $\mathrm{C}_{13} \mathrm{H}_{11} \mathrm{ClFN}_{3} \mathrm{O}[\mathrm{M}+\mathrm{H}]^{+} 280.06474$, found 280.06392 . 


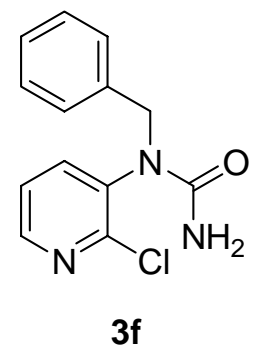

1-Benzyl-1-(2-chloro-pyridin-3-yl)urea (3f): Following general procedure A, 3f was obtained as an off-white solid (86\%): ${ }^{1} \mathrm{H}$ NMR (400 MHz, DMSO) $\delta 3.91-5.64(\mathrm{br}, 2 \mathrm{H})$, $6.02(\mathrm{~s}, 2 \mathrm{H}), 7.10-7.32(\mathrm{~m}, 6 \mathrm{H}), 7.41(\mathrm{~d}, J=7.6,1.2 \mathrm{~Hz}, 1 \mathrm{H}), 8.25(\mathrm{~d}, J=4.4,1.2 \mathrm{~Hz}$, $1 \mathrm{H}) ;{ }^{13} \mathrm{C}\left\{{ }^{1} \mathrm{H}\right\}$ NMR (100 MHz, DMSO) $\delta 51.6,124.3,127.7,128.7$ (2C), 136.4, 138.4, 140.9, 148.7, 150.6, 157.3. HRMS calcd. for $\mathrm{C}_{13} \mathrm{H}_{12} \mathrm{ClN}_{3} \mathrm{O}[\mathrm{M}+\mathrm{H}]^{+}$262.07417, found 262.07341 .

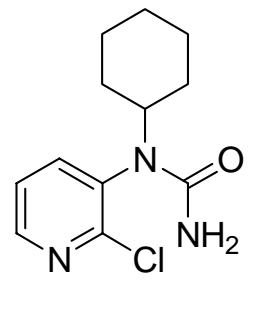

$3 g$

1-(2-Chloro-pyridin-3-yl)-1-cyclohexylurea (3g): Following general procedure A, 3g was obtained as an off-white solid (86\%): ${ }^{1} \mathrm{H}$ NMR (400 MHz, DMSO) $\delta$ 0.75-0.98 (m, $2 \mathrm{H}), 1.03-1.21(\mathrm{~m}, 1 \mathrm{H}), 1.25-1.43(\mathrm{~m}, 2 \mathrm{H}), 1.51-1.61(\mathrm{~m}, 1 \mathrm{H}), 1.63-1.78(\mathrm{~m}, 2 \mathrm{H}), 1.91-$ 2.07 (m, 2H), 4.32 (tt, $J=12.4,3.6 \mathrm{~Hz}, 1 \mathrm{H}), 4.53$ (s, 2H), 7.33 (dd, $J=8.0,4.4 \mathrm{~Hz}, 1 \mathrm{H}$ ), $7.61(\mathrm{dd}, J=8.0,2.0 \mathrm{~Hz}, 1 \mathrm{H}), 8.40(\mathrm{dd}, J=4.4,2.0 \mathrm{~Hz}, 1 \mathrm{H}) ;{ }^{13} \mathrm{C}\left\{{ }^{1} \mathrm{H}\right\} \mathrm{NMR}(100 \mathrm{MHz}$, DMSO) $\delta 25.3,25.8,30.5$ and 33.0 (br, rotamers), 56.6, 123.1, 133.8, 141.0, 149.1, 153.0, 156.4. HRMS calcd. for $\mathrm{C}_{12} \mathrm{H}_{16} \mathrm{ClN}_{3} \mathrm{O}[\mathrm{M}+\mathrm{H}]^{+} 254.10547$, found 254.10667. 


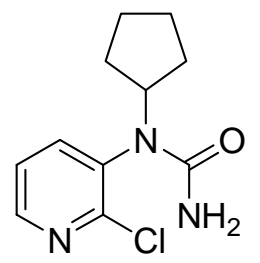

3h

1-(2-Chloro-pyridin-3-yl)-1-cyclopentylurea (3h): Following general procedure A, 3h was obtained as an off-white solid (83\%): ${ }^{1} \mathrm{H}$ NMR (400 MHz, DMSO) $\delta ~ 0.81-1.32$ (br m, 2H), 1.32-1.50 (m, 4H), 1.67-1.95 (br m, 2H), 4.34-4.46 (m, 1H), 5.77 (s, 2H), 7.42 $(\mathrm{dd}, J=7.6,4.4 \mathrm{~Hz}, 1 \mathrm{H}), 7.72(\mathrm{dd}, J=7.6,2.0 \mathrm{~Hz}, 1 \mathrm{H}), 8.33(\mathrm{dd}, J=4.4,2.0,1 \mathrm{H}) ;{ }^{13} \mathrm{C}$ $\left\{{ }^{1} \mathrm{H}\right\}$ NMR (100 MHz, DMSO) $\delta$ 22.6, 30.0 (br), 58.3, 124.4, 134.7, 142.3, 148.9, 152.5, 157.2. HRMS calcd. for $\mathrm{C}_{11} \mathrm{H}_{14} \mathrm{ClN}_{3} \mathrm{O}[\mathrm{M}+\mathrm{H}]^{+} 240.08982$, found 240.08965 .

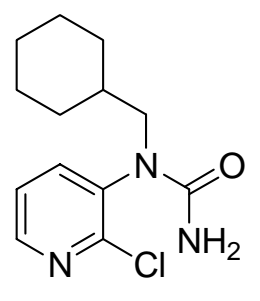

3i

1-(2-Chloro-pyridin-3-yl)-1-cyclohexylmethylurea (3i): Following general procedure A, 3i was obtained as an off-white solid (85\%): ${ }^{1} \mathrm{H}$ NMR (400 MHz, DMSO) $\delta$ 0.74-0.93 (m, 2H), 0.99-1.17 (m, 3H), 1.20-1.35 (m, 1H), 1.45-1.74 (m, 5H), $3.29(\mathrm{~s}, 2 \mathrm{H}), 5.82(\mathrm{~s}$, 2H), $7.43(\mathrm{dd}, J=8.0,4.2 \mathrm{~Hz}, 1 \mathrm{H}), 7.77$ (dd, $J=8.0,2.0 \mathrm{~Hz}, 1 \mathrm{H}), 8.30$ (dd, $J=4.8,2.0$ $\mathrm{Hz}, 1 \mathrm{H}) ;{ }^{13} \mathrm{C}\left\{{ }^{1} \mathrm{H}\right\}$ NMR (100 MHz, DMSO) $\delta 25.9,26.5,30.8,37.1,54.5,124.6,137.5$, 141.1, 148.4, 150.5, 157.4. HRMS calcd. for $\mathrm{C}_{13} \mathrm{H}_{18} \mathrm{ClN}_{3} \mathrm{O}[\mathrm{M}+\mathrm{H}]^{+}$268.12112, found 268.1217. 


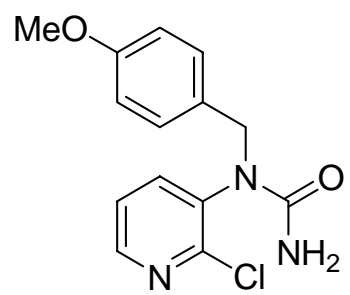

3j

1-(2-Chloro-pyridin-3-yl)-1-(4-methoxy-benzyl)urea (3j): Following general procedure A, 3j was obtained as an off-white solid (\%): ${ }^{1} \mathrm{H}$ NMR (400 MHz, DMSO) $\delta$ 3.66 (s, 3H), 3.80-5.35 (br, 2H), 5.98 (br s, 2H), 6.77 (d, J=8.4 Hz, 2H), 7.07 (d, $J=8.4$ $\mathrm{Hz}, 2 \mathrm{H}), 7.28(\mathrm{dd}, J=8.0,4.8 \mathrm{~Hz}, 1 \mathrm{H}), 7.35(\mathrm{dd}, J=8.0,2.0 \mathrm{~Hz}, 1 \mathrm{H}), 8.24(\mathrm{dd}, J=4.8$, $2.0 \mathrm{~Hz}, 1 \mathrm{H}) ;{ }^{13} \mathrm{C}\left\{{ }^{1} \mathrm{H}\right\} \mathrm{NMR}(100 \mathrm{MHz}, \mathrm{DMSO}) \delta 50.9,55.5,114.1,124.3,130.2(2 \mathrm{C})$, 136.3, 141.1, 148.6, 150.7, 157.3, 158.9. HRMS calcd. for $\mathrm{C}_{14} \mathrm{H}_{14} \mathrm{ClN}_{3} \mathrm{O}_{2}[\mathrm{M}+\mathrm{H}]^{+}$ 292.08473, found 292.08587.

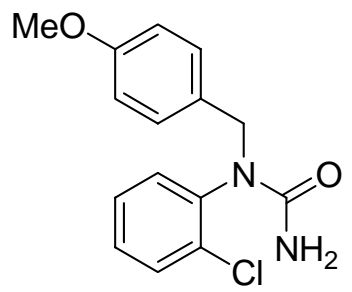

3k

1-(2-Chloro-phenyl)-1-(4-methoxy-benzyl)urea (3k): Following general procedure A, 3k was obtained as an off-white solid (85\%): ${ }^{1} \mathrm{H}$ NMR (400 MHz, DMSO) $\delta 3.69$ (s, 3H), 3.89-4.59 (br, 1H), 4.65-5.35 (br, 1H), 5.69 (s, 2H), 6.80 (d, J = 8.4 Hz, 2H), 6.96 (dd, $J$ $=8.0,2.0 \mathrm{~Hz}, 1 \mathrm{H}), 7.09(\mathrm{~d}, J=8.4 \mathrm{~Hz}, 2 \mathrm{H}), 7.19-7.31(\mathrm{~m}, 2 \mathrm{H}), 7.51(\mathrm{dd}, J=8.0,1.2 \mathrm{~Hz}$, $1 \mathrm{H}) ;{ }^{13} \mathrm{C}\left\{{ }^{1} \mathrm{H}\right\} \mathrm{NMR}(100 \mathrm{MHz}, \mathrm{DMSO}) \delta$ 51.0, 55.3, 113.9, 128.3, 129.5, 129.9, 130.6, 130.7, 132.0, 133.2, 139.3, 157.4, 158.7. HRMS calcd. for $\mathrm{C}_{15} \mathrm{H}_{15} \mathrm{ClN}_{2} \mathrm{O}_{2}[\mathrm{M}+\mathrm{H}]^{+}$ 291.0900, found 291.0904. 


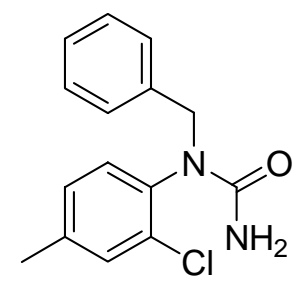

$3 \mid$

1-Benzyl-1-(2-chloro-4-methyl-phenyl)urea (3l): Following general procedure A, 31 was obtained as an off-white solid (80\%): ${ }^{1} \mathrm{H}$ NMR (400 MHz, DMSO) $\delta 2.26(\mathrm{~s}, 3 \mathrm{H})$, 3.88-4.38 (br, 1H), 4.96-5.38 (br, 1H), 5.69 (br s, 2H), 6.86 (d, $J=8.0 \mathrm{~Hz}, 1 \mathrm{H}), 7.01$ (d, $J$ $=8.0 \mathrm{~Hz}, 1 \mathrm{H}), 7.12-7.29(\mathrm{~m}, 5 \mathrm{H}), 7.34(\mathrm{~s}, 1 \mathrm{H}) ;{ }^{13} \mathrm{C}\left\{{ }^{1} \mathrm{H}\right\}$ NMR $(100 \mathrm{MHz}, \mathrm{DMSO}) \delta$ 20.7, 51.6, 127.3, 128.5, 128.6, 129.0, 131.0, 131.5, 132.7, 136.6, 138.8, 139.4, 157.6. HRMS calcd. for $\mathrm{C}_{15} \mathrm{H}_{15} \mathrm{ClN}_{2} \mathrm{O}[\mathrm{M}+\mathrm{H}]^{+} 275.09457$, found 275.09479 .

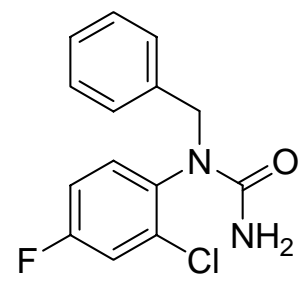

$3 m$

1-Benzyl-1-(2-chloro-4-fluoro-phenyl)urea (3m): Following general procedure A, 3m was obtained as an off-white solid (81\%): ${ }^{1} \mathrm{H}$ NMR (400 MHz, DMSO) $\delta 3.97-4.35$ (br, 1H), 5.01-5.31 (br, 1H), 5.83 (br s, 2H), 7.00 (dd, $J=8.4,6.0 \mathrm{~Hz}, 1 \mathrm{H}$ ), 7.10 (dd, $J=8.4$, $2.8 \mathrm{~Hz}, 1 \mathrm{H}), 7.17-7.29(\mathrm{~m}, 5 \mathrm{H}), 7.50(\mathrm{dd}, J=8.4,2.8 \mathrm{~Hz}, 1 \mathrm{H}) ;{ }^{13} \mathrm{C}\left\{{ }^{1} \mathrm{H}\right\}$ NMR $(100$ MHz, DMSO) $\delta 51.6,115.4\left(\mathrm{~d}, J_{\mathrm{CF}}=20.1 \mathrm{~Hz}\right), 118.0\left(\mathrm{~d}, J_{\mathrm{CF}}=30.2 \mathrm{~Hz}\right), 127.4,128.5$, $128.6,133.3\left(\mathrm{~d}, J_{\mathrm{CF}}=10.1 \mathrm{~Hz}\right), 134.5\left(\mathrm{~d}, J_{\mathrm{CF}}=10.1 \mathrm{~Hz}\right), 135.9,138.5,157.5,161.2(\mathrm{~d}$, $J_{\mathrm{CF}}=251.5 \mathrm{~Hz}$ ). HRMS calcd. for $\mathrm{C}_{14} \mathrm{H}_{12} \mathrm{ClFN}_{2} \mathrm{O}[\mathrm{M}+\mathrm{H}]^{+} 279.06950$, found 279.07002 . 


\section{General procedure B}

Pd-Catalyzed urea cyclization with dppb: To a Morton-type flask equipped with stirbar, reflux condenser and temperature probe was charged $\mathrm{NaHCO}_{3}$ (300 mol\%), urea (100 mol\%), and $\mathrm{i}-\mathrm{PrOH}\left(750-1500 \mathrm{ppm} \mathrm{H}_{2} \mathrm{O}, 10 \mathrm{~mL} / \mathrm{g}\right.$ substrate). The heterogeneous mixture was agitated and purged with $\mathrm{N}_{2}$ using an $\mathrm{M}$-fritted gas dispersion tube. After 30 $\min , \mathrm{Pd}(\mathrm{OAc})_{2}(1 \mathrm{~mol} \%)$ and $\mathrm{dppb}(2 \mathrm{~mol} \%)$ were added and the $\mathrm{N}_{2}$ purge continued for a further $30 \mathrm{~min}$. The resulting pink mixture was heated to $83{ }^{\circ} \mathrm{C}$ (reflux) for $24 \mathrm{~h}$. After this time, LC analysis of the yellow mixture indicated $>99 \%$ conversion. Atmospheric pressure distillation was initiated and continued until $\sim 60 \%$ of $i$-PrOH had been removed. $i$-PrOAc was added to achieve an $\sim 2: 1$ ratio of $i$-PrOAc $: i$-PrOH (the $i$-PrOH aids solubility during workup). Water ( $3 \mathrm{~mL} / \mathrm{g}$ substrate) was added and the temperature was maintained at $60^{\circ} \mathrm{C}$ throughout workup to ensure solubility. After 15 min agitation, the separated organic phase was washed with brine. Azeotropic drying with $i$-PrOAc was conducted at atmospheric pressure under constant volume conditions until the water content was $<150$ ppm measured by KF titration ( $i-\mathrm{PrOH}$ was also removed during this process). The product was isolated by crystallization.

\section{General procedure C}

Pd-Catalyzed urea cyclization with X-Phos: To a Morton-type flask equipped with stirbar, reflux condenser and temperature probe was charged $\mathrm{NaHCO}_{3}$ (300 mol\%), urea (100 mol\%), and $\mathrm{i}-\mathrm{PrOH}\left(750-1500 \mathrm{ppm} \mathrm{H}_{2} \mathrm{O}, 10 \mathrm{~mL} / \mathrm{g}\right.$ substrate). The heterogeneous mixture was agitated and purged with $\mathrm{N}_{2}$ using an $\mathrm{M}$-fritted gas dispersion tube. After 30 min, $\mathrm{Pd}(\mathrm{OAc})_{2}(1 \mathrm{~mol} \%)$ and $\mathrm{X}-\mathrm{Phos}(3 \mathrm{~mol} \%)$ were added and the $\mathrm{N}_{2}$ purge continued for a further $30 \mathrm{~min}$. The resulting mixture was heated to $83{ }^{\circ} \mathrm{C}$ (reflux) for $24 \mathrm{~h}$. After this time, LC analysis of the yellow mixture indicated $>99 \%$ conversion. Atmospheric pressure distillation was initiated and continued until $\sim 60 \%$ of $i$-PrOH had been removed. $i$-PrOAc was added to achieve an $\sim 2: 1$ ratio of $i$-PrOAc $: i$-PrOH (the $i$-PrOH aids solubility during workup). Water ( $3 \mathrm{~mL} / \mathrm{g}$ substrate) was added and the temperature was maintained at $60{ }^{\circ} \mathrm{C}$ throughout workup to ensure solubility. After 15 min agitation, the separated organic phase was washed with brine. Azeotropic drying with $i$-PrOAc was conducted at atmospheric pressure under constant volume conditions until the water 
content was $<150 \mathrm{ppm}$ measured by KF titration (i-PrOH was also removed during this process). The product was isolated by crystallization.

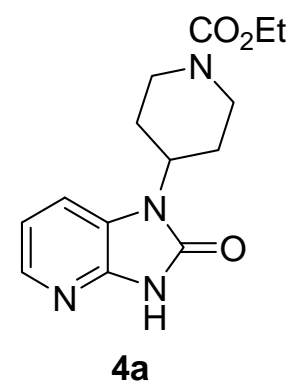

4-(2-Oxo-2,3-dihydroimidazo[4,5-b]pyridin-1-yl)piperidine-1-carboxylic acid ethyl ester (4a): Following general procedure B, 4a was obtained as an off-white solid (96\%): ${ }^{1} \mathrm{H}$ NMR (400 MHz, DMSO) $\delta 1.18$ (t, $\left.J=7.2 \mathrm{~Hz}, 3 \mathrm{H}\right), 1.65-1.76(\mathrm{~m}, 2 \mathrm{H}), 2.06-2.22$ (m, 2H), 2.78-3.03 (br m, 2H), 4.04 (q, J = 7.2 Hz, 2H), 4.08-4.20 (m, 2H), 4.30-4.41 (m, 1H), $6.95(\mathrm{dd}, J=7.6,5.2 \mathrm{~Hz}, 1 \mathrm{H}), 7.54(\mathrm{dd}, J=7.6,1.2 \mathrm{~Hz}, 1 \mathrm{H}), 7.87$ (dd, $J=5.2,1.2$ $\mathrm{Hz}, 1 \mathrm{H}), 11.47$ (br, $1 \mathrm{H}) ;{ }^{13} \mathrm{C}\left\{{ }^{1} \mathrm{H}\right\}$ NMR (100 MHz, DMSO) $\delta$ 14.9, 29.0, 43.5, 50.2, 61.1, 115.0, 116.7, 123.6, 140.0, 143.8, 153.5, 154.9. HRMS calcd. for $\mathrm{C}_{14} \mathrm{H}_{18} \mathrm{~N}_{4} \mathrm{O}_{3}$ $[\mathrm{M}+\mathrm{H}]^{+}$291.14517, found 291.14598.

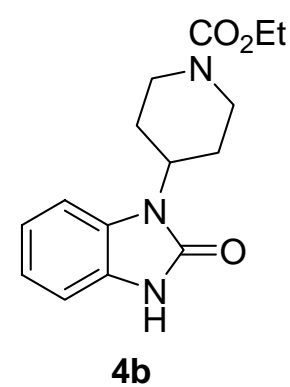

4-(2-Oxo-2,3-dihydrobenzoimidazol-1-yl)piperidine-1-carboxylic acid ethyl ester (4b) $)^{2}$ : Following general procedure C, $\mathbf{4 b}$ was obtained as an off-white solid (89\%): ${ }^{1} \mathrm{H}$ NMR (400 MHz, DMSO) $\delta 1.20(\mathrm{t}, J=7.2 \mathrm{~Hz}, 3 \mathrm{H}), 1.62-1.73(\mathrm{~m}, 2 \mathrm{H}), 2.12-2.28$ (m,

\footnotetext{
${ }^{2}$ Gustin, D. J.; Sehon, C. A.; Wei, J.; Cai, H.; Meduna, S. P.; Khatuya, H.; Sun, S.; Gu, Y.; Jiang, W.; Thurmond, R. L.; Karlsson, L.; Edwards, J. P. Biorog. Med. Chem. Lett. 2005, 15, 1687-1692.
} 
2H), 2.78-3.03 (br m, 2H), 4.07 (q, J = 7.2 Hz, 2H), 4.09-4.18 (m, 2H), 4.27-4.40 (m, 1H), 6.93-6.99 (m, 3H), 7.18-7.25 (m, 1H), $10.82(\mathrm{~s}, 1 \mathrm{H}) ;{ }^{13} \mathrm{C}\left\{{ }^{1} \mathrm{H}\right\}$ NMR $(100 \mathrm{MHz}$, DMSO) $\delta$ 14.9, 28.9, 43.4, 50.2, 61.1, 108.8, 109.2, 120.7, 120.9, 128.7, 129.6, 154.0, 154.9. HRMS calcd. for $\mathrm{C}_{15} \mathrm{H}_{19} \mathrm{~N}_{3} \mathrm{O}_{3}[\mathrm{M}+\mathrm{H}]^{+}$290.14992, found 290.14957 .

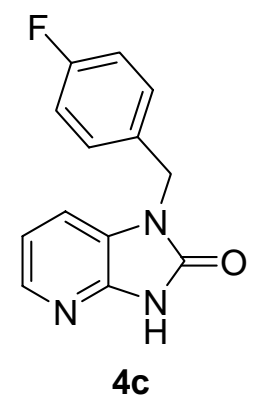

1-(4-Fluoro-benzyl)-1,3-dihydroimidazo[4,5-b]pyridin-2-one (4c): Following general procedure B, 4c was obtained as an off-white solid (95\%): ${ }^{1} \mathrm{H}$ NMR (400 MHz, DMSO) $\delta$ $5.00(\mathrm{~s}, 2 \mathrm{H}), 6.96(\mathrm{dd}, J=8.0,5.2 \mathrm{~Hz}, 1 \mathrm{H}), 7.11-7.19(\mathrm{~m}, 2 \mathrm{H}), 7.34-7.41(\mathrm{~m}, 3 \mathrm{H}), 7.89$ (dd, $J=5.2,1.6 \mathrm{~Hz}, 1 \mathrm{H}), 11.64(\mathrm{~s}, 1 \mathrm{H}) ;{ }^{13} \mathrm{C}\left\{{ }^{1} \mathrm{H}\right\}$ NMR (100 MHz, DMSO) $\delta 42.8$, $114.4,115.8\left(\mathrm{~d}, J_{\mathrm{CF}}=30.2 \mathrm{~Hz}\right), 117.0,124.3,130.0\left(\mathrm{~d}, J_{\mathrm{CF}}=10.1 \mathrm{~Hz}\right), 133.3,140.5$, 143.9, 154.1, $161.9\left(\mathrm{~d}, J_{\mathrm{CF}}=241.5 \mathrm{~Hz}\right)$. HRMS calcd. for $\mathrm{C}_{13} \mathrm{H}_{10} \mathrm{FN}_{3} \mathrm{O}[\mathrm{M}+\mathrm{H}]^{+}$ 244.08807, found 244.08968 .

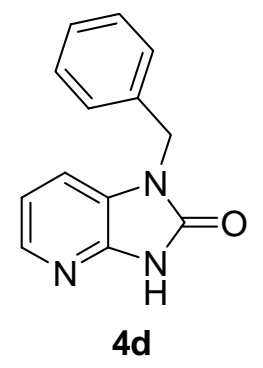

1-Benzyl-1,3-dihydroimidazo[4,5-b]pyridin-2-one (4d) ${ }^{3}$ : Following general procedure B, 4d was obtained as an off-white solid (95\%): ${ }^{1} \mathrm{H}$ NMR (400 MHz, DMSO) $\delta 5.01$ (s, $2 \mathrm{H}), 6.92(\mathrm{dd}, J=8.0,5.6 \mathrm{~Hz}, 1 \mathrm{H}), 7.18-7.26(\mathrm{~m}, 1 \mathrm{H}), 7.27-7.34(\mathrm{~m}, 5 \mathrm{H}), 7.89$ (d, $J=5.6$

\footnotetext{
${ }^{3}$ Meanwell, N.; Sit, S. Y.; Gao, J.; Wong, H. S.; Gao, Q.; St. Laurent, D. R.; Balasubramanian, N. J. Org. Chem. 1995, 60, 1565-1582.
} 
$\mathrm{Hz}, 1 \mathrm{H}), 11.68(\mathrm{~s}, 1 \mathrm{H}) ;{ }^{13} \mathrm{C}\left\{{ }^{1} \mathrm{H}\right\} \mathrm{NMR}(100 \mathrm{MHz}, \mathrm{DMSO}) \delta 43.5,114.4,117.0,124.4$, 127.8, 127.9, 129.0, 137.1, 140.5, 143.9, 154.1. HRMS calcd. for $\mathrm{C}_{13} \mathrm{H}_{11} \mathrm{~N}_{3} \mathrm{O}[\mathrm{M}+\mathrm{H}]^{+}$ 226.09749 , found 226.09812 .

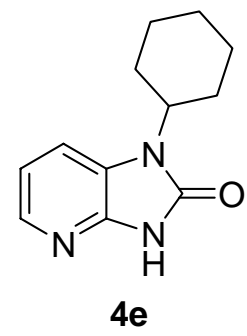

1-Cyclohexyl-1,3-dihydroimidazo[4,5-b]pyridin-2-one (4e): Following general procedure B, 4e was obtained as an off-white solid (93\%): ${ }^{1} \mathrm{H}$ NMR (400 MHz, DMSO) $\delta$ 1.15-1.43 (m, 3H), 1.56-1.73 (m, 3H), 1.74-1.85 (m, 2H), 1.91-2.08 (m, 2H), 4.06-4.19 (m, 1H), $6.94(\mathrm{dd}, J=8.0,5.2 \mathrm{~Hz}, 1 \mathrm{H}), 7.56(\mathrm{dd}, J=8.0,1.2 \mathrm{~Hz}, 1 \mathrm{H}), 7.86(\mathrm{dd}, J=5.2$, $1.2 \mathrm{~Hz}, 1 \mathrm{H}), 11.49(\mathrm{~s}, 1 \mathrm{H}) ;{ }^{13} \mathrm{C}\left\{{ }^{1} \mathrm{H}\right\}$ NMR (100 MHz, DMSO) $\delta$ 25.0, 25.8, 29.9, 51.8, $115.2,116.6,123.5,139.8,143.8,153.4$. HRMS calcd. for $\mathrm{C}_{12} \mathrm{H}_{15} \mathrm{~N}_{3} \mathrm{O}[\mathrm{M}+\mathrm{H}]^{+}$ 218.12879 , found 218.13001 .

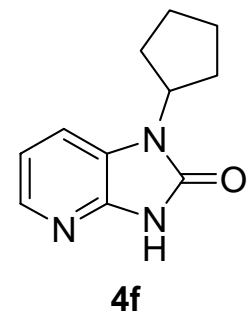

1-Cyclopentyl-1,3-dihydroimidazo[4,5-b]pyridin-2-one (4f): Following general procedure B, 4f was obtained as an off-white solid (94\%): ${ }^{1} \mathrm{H}$ NMR (400 MHz, DMSO) $\delta$ 1.54-1.67 (m, 2H), 1.77-2.02 (6H), 4.62-4.75 (m, 1H), $6.96(\mathrm{dd}, J=8.0,5.2 \mathrm{~Hz}, 1 \mathrm{H})$, $7.42(\mathrm{~d}, J=8.0 \mathrm{~Hz}, 1 \mathrm{H}), 7.88(\mathrm{~d}, J=5.2 \mathrm{~Hz}, 1 \mathrm{H}), 11.49(\mathrm{~s}, 1 \mathrm{H}) ;{ }^{13} \mathrm{C}\left\{{ }^{1} \mathrm{H}\right\}$ NMR $(100$ MHz, DMSO) $\delta$ 24.7, 28.7, 52.6, 114.8, 116.7, 123.3, 139.9, 143.9, 153.6. HRMS calcd. for $\mathrm{C}_{11} \mathrm{H}_{13} \mathrm{~N}_{3} \mathrm{O}[\mathrm{M}+\mathrm{H}]^{+}$204.11314, found 204.11401. 


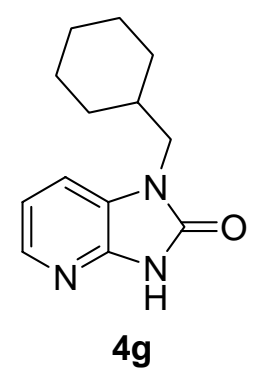

1-Cyclohexylmethyl-1,3-dihydroimidazo[4,5-b]pyridin-2-one (4g) ${ }^{3}$ : Following general procedure B, $\mathbf{4 g}$ was obtained as an off-white solid (95\%): ${ }^{1} \mathrm{H}$ NMR $(400 \mathrm{MHz}, \mathrm{DMSO})$ $\delta$ 0.84-0.99 (m, 2H), 1.02-1.17 (m, 3H), 1.45-1.64 (m, 5H), 1.66-1.79 (m, 1H), 3.57 (d, $J$ $=7.6 \mathrm{~Hz}, 2 \mathrm{H}), 6.95(\mathrm{dd}, J=8.0,4.8 \mathrm{~Hz}, 1 \mathrm{H}), 7.39$ (d, $J=8.0 \mathrm{~Hz}, 1 \mathrm{H}), 7.84(\mathrm{~d}, J=4.8$ $\mathrm{Hz}, 1 \mathrm{H}), 11.45(\mathrm{~s}, 1 \mathrm{H}) ;{ }^{13} \mathrm{C}\left\{{ }^{1} \mathrm{H}\right\}$ NMR (100 MHz, DMSO) $\delta$ 25.6, 26.3, 30.6, 37.1, 46.3, 114.4, 117.0, 125.3, 140.2, 143.9, 154.3. HRMS calcd. for $\mathrm{C}_{13} \mathrm{H}_{17} \mathrm{~N}_{3} \mathrm{O}[\mathrm{M}+\mathrm{H}]^{+}$ 232.14444 , found 232.14495 .

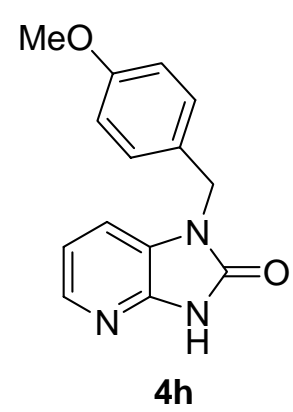

1-(4-Methoxy-benzyl)-1,3-dihydroimidazo[4,5-b]pyridin-2-one $\quad$ (4h): $\quad$ Following general procedure B, $\mathbf{4 h}$ was obtained as an off-white solid (95\%): ${ }^{1} \mathrm{H}$ NMR $(400 \mathrm{MHz}$, DMSO) $\delta 3.70(\mathrm{~s}, 3 \mathrm{H}), 4.92(\mathrm{~s}, 2 \mathrm{H}), 6.88(\mathrm{~d}, J=8.0 \mathrm{~Hz}, 2 \mathrm{H}), 6.94(\mathrm{dd}, J=8.0,5.2 \mathrm{~Hz}$, $1 \mathrm{H}), 7.27(\mathrm{~d}, J=8.0 \mathrm{~Hz}, 2 \mathrm{H}), 7.34(\mathrm{dd}, J=8.0,1.2 \mathrm{~Hz}, 1 \mathrm{H}), 7.88(\mathrm{dd}, J=5.2,1.2 \mathrm{~Hz}$, $1 \mathrm{H}), 11.60(\mathrm{~s}, 1 \mathrm{H}) ;{ }^{13} \mathrm{C}\left\{{ }^{1} \mathrm{H}\right\}$ NMR (100 MHz, DMSO) $\delta$ 43.0, 55.4, 114.4, 114.1, 116.9, 124.3, 129.0, 129.3, 140.4, 143.9, 154.1, 159.0. HRMS calcd. for $\mathrm{C}_{14} \mathrm{H}_{13} \mathrm{~N}_{3} \mathrm{O}_{2}[\mathrm{M}+\mathrm{H}]^{+}$ 256.1086 , found 256.1087 . 


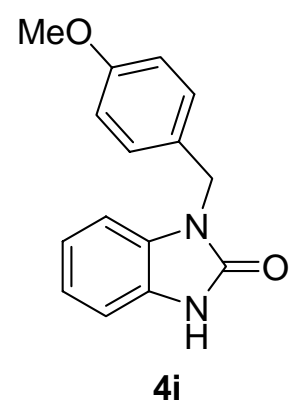

1-(4-Methoxy-benzyl)-1,3-dihydrobenzoimidazol-2-one (4i): Following general procedure C, 4i was obtained as an off-white solid (92\%): ${ }^{1} \mathrm{H}$ NMR (400 MHz, DMSO) $\delta$ $3.68(\mathrm{~s}, 3 \mathrm{H}), 4.91(\mathrm{~s}, 2 \mathrm{H}), 6.86(\mathrm{~d}, \mathrm{~J}=8.0 \mathrm{~Hz}, 2 \mathrm{H}), 6.91-7.04(\mathrm{~m}, 4 \mathrm{H}), 7.26(\mathrm{~d}, \mathrm{~J}=8.0$ $\mathrm{Hz}, 2 \mathrm{H}), 10.91(\mathrm{br}, 1 \mathrm{H}) ;{ }^{13} \mathrm{C}\left\{{ }^{1} \mathrm{H}\right\}$ NMR (100 MHz, DMSO) $\delta$ 43.0, 55.4, 108.4, 109.2, $114.3,120.8,121.3,128.7,129.2,129.5,130.3,154.7,158.9$. HRMS calcd. for $\mathrm{C}_{15} \mathrm{H}_{14} \mathrm{~N}_{4} \mathrm{O}_{2}[\mathrm{M}+\mathrm{H}]^{+}$255.1134, found 255.1130.

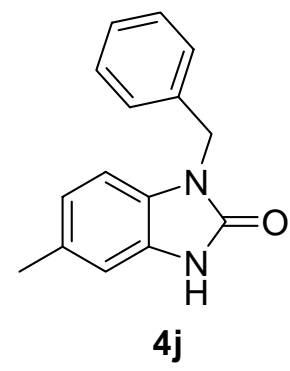

1-Benzyl-5-methyl-1,3-dihydrobenzoimidazol-2-one $\quad(\mathbf{4 j})^{4}: \quad$ Following general procedure $\mathrm{C}, \mathbf{4} \mathbf{j}$ was obtained as an off-white solid $(90 \%)$ : ${ }^{1} \mathrm{H}$ NMR (400 MHz, DMSO) $\delta$ 2.23 (s, 3H), 4.93 (s, 2H), 6.70 (d, $J=8.0 \mathrm{~Hz}, 1 \mathrm{H}), 6.78$ (s, 1H), 6.83 (d, $J=8.0 \mathrm{~Hz}, 1 \mathrm{H}$ ), 7.15-7.22 (m, $J=8.0 \mathrm{~Hz}, 1 \mathrm{H}), 7.23-7.31(\mathrm{~m}, 4 \mathrm{H}), 10.85(\mathrm{br}, 1 \mathrm{H}) ;{ }^{13} \mathrm{C}\left\{{ }^{1} \mathrm{H}\right\}$ NMR $(100$ MHz, DMSO) $\delta$ 21.4, 43.7, 108.3, 109.9, 121.5, 127.7, 127.8, 128.4, 128.9, 129.0, 130.6, 137.8, 155.0. HRMS calcd. for $\mathrm{C}_{15} \mathrm{H}_{14} \mathrm{~N}_{2} \mathrm{O}[\mathrm{M}+\mathrm{H}]^{+} 239.11789$, found 239.11756 .

\footnotetext{
${ }^{4}$ Kametani, T.; Shio, M. J. Heterocyclic Chem. 1970, 7, 831-834.
} 


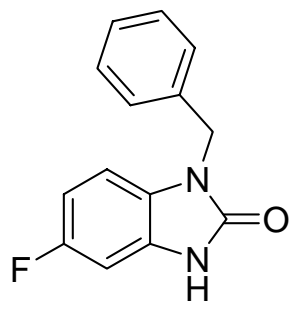

$4 \mathbf{k}$

1-Benzyl-5-fluoro-1,3-dihydrobenzoimidazol-2-one $\quad(\mathbf{4 k})^{5}: \quad$ Following general procedure C, 4k was obtained as an off-white solid (91\%): ${ }^{1} \mathrm{H}$ NMR (400 MHz, DMSO) $\delta 4.96(\mathrm{~s}, 2 \mathrm{H}), 6.73(\mathrm{dd}, J=8.8,2.4 \mathrm{~Hz}, 1 \mathrm{H}), 6.83(\mathrm{dd}, J=8.8,2.4 \mathrm{~Hz}, 1 \mathrm{H}), 6.94(\mathrm{dd}, J=$ 8.8, $4.8 \mathrm{~Hz}, 1 \mathrm{H}), 7.16-7.24(\mathrm{~m}, 1 \mathrm{H}), 7.25-7.30(\mathrm{~m}, 4 \mathrm{H}), 11.10(\mathrm{br}, 1 \mathrm{H}) ;{ }^{13} \mathrm{C}\left\{{ }^{1} \mathrm{H}\right\} \mathrm{NMR}$ $(100 \mathrm{MHz}, \mathrm{DMSO}) \delta 43.8,97.5\left(\mathrm{~d}, \mathrm{~J}_{\mathrm{CF}}=28.2 \mathrm{~Hz}\right), 107.2\left(\mathrm{~d}, J_{\mathrm{CF}}=24.1 \mathrm{~Hz}\right), 108.9\left(\mathrm{~d}, J_{\mathrm{CF}}\right.$ $=9.0 \mathrm{~Hz}), 126.9,127.8,127.9,129.1,129.5\left(\mathrm{~d}, J_{\mathrm{CF}}=13.1 \mathrm{~Hz}\right), 137.5,155.2,158.3(\mathrm{~d}$, $J_{\mathrm{CF}}=234.4 \mathrm{~Hz}$ ). HRMS calcd. for $\mathrm{C}_{14} \mathrm{H}_{11} \mathrm{FN}_{2} \mathrm{O}[\mathrm{M}+\mathrm{H}]^{+}$243.09282, found 243.09200.

\footnotetext{
${ }^{5}$ Orjales, A.; Mosquera, R.; Labeaga, L.; Rodes, R. J. Med. Chem. 1997, 40, 586-593.
} 

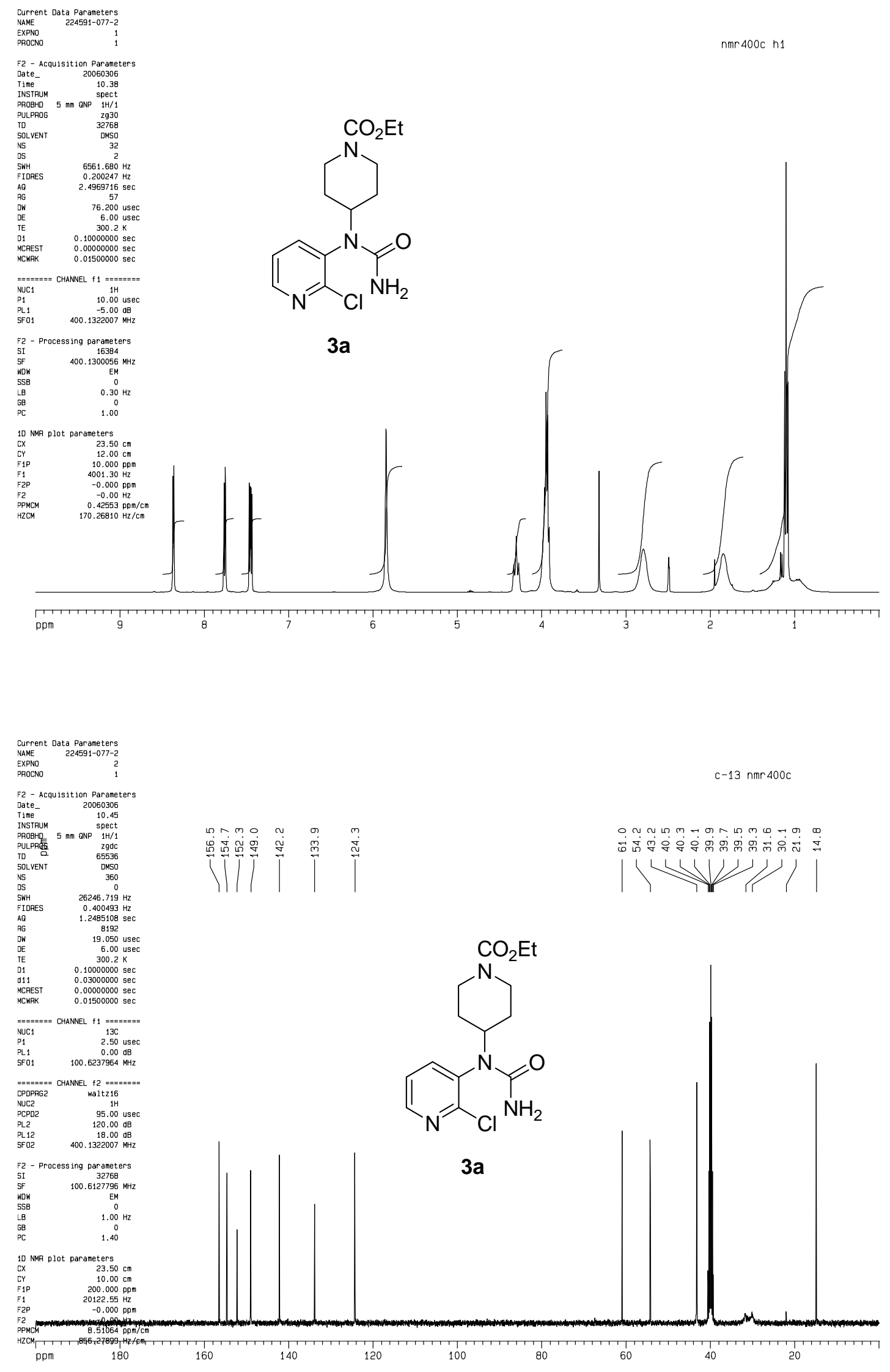

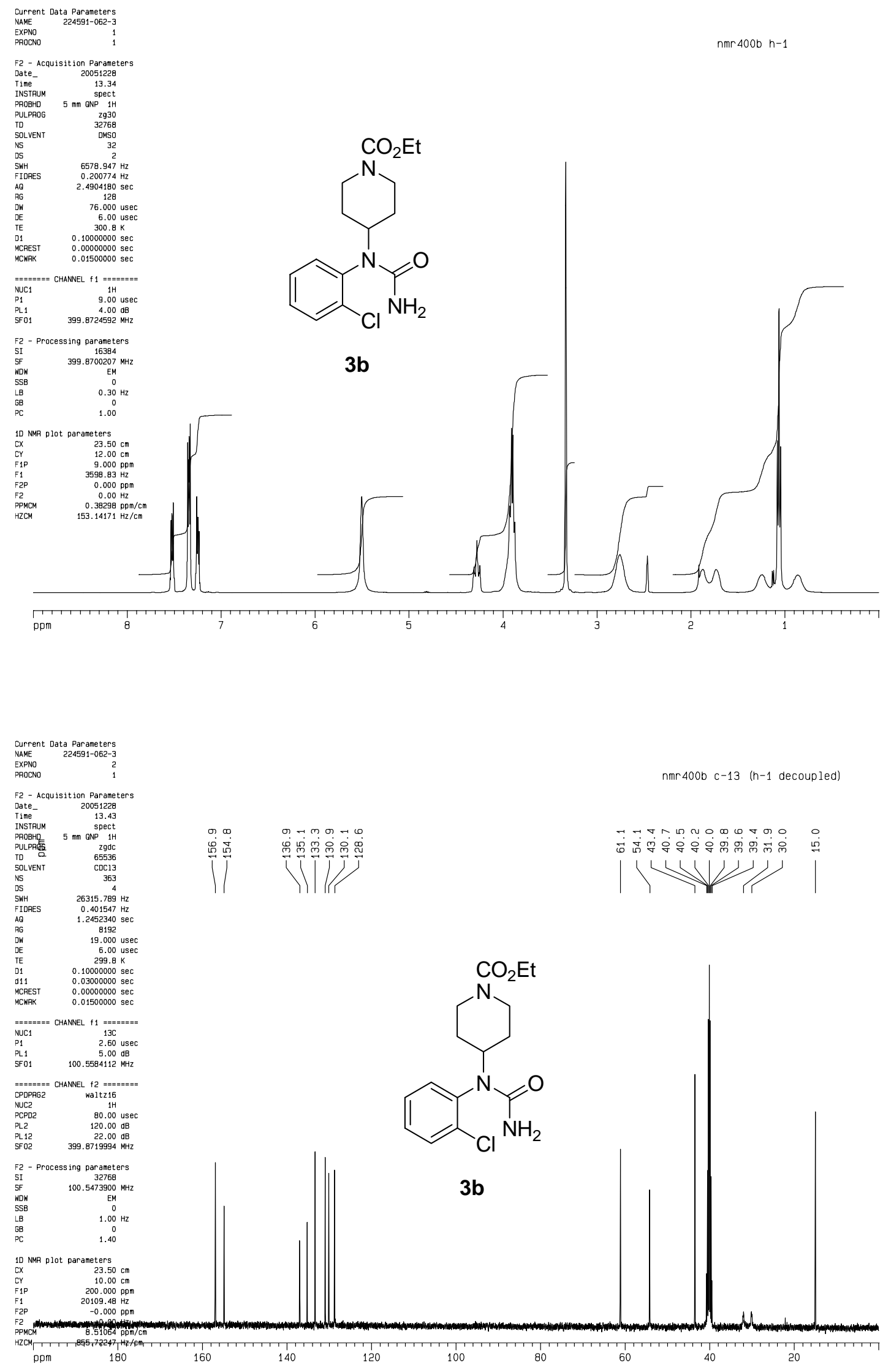


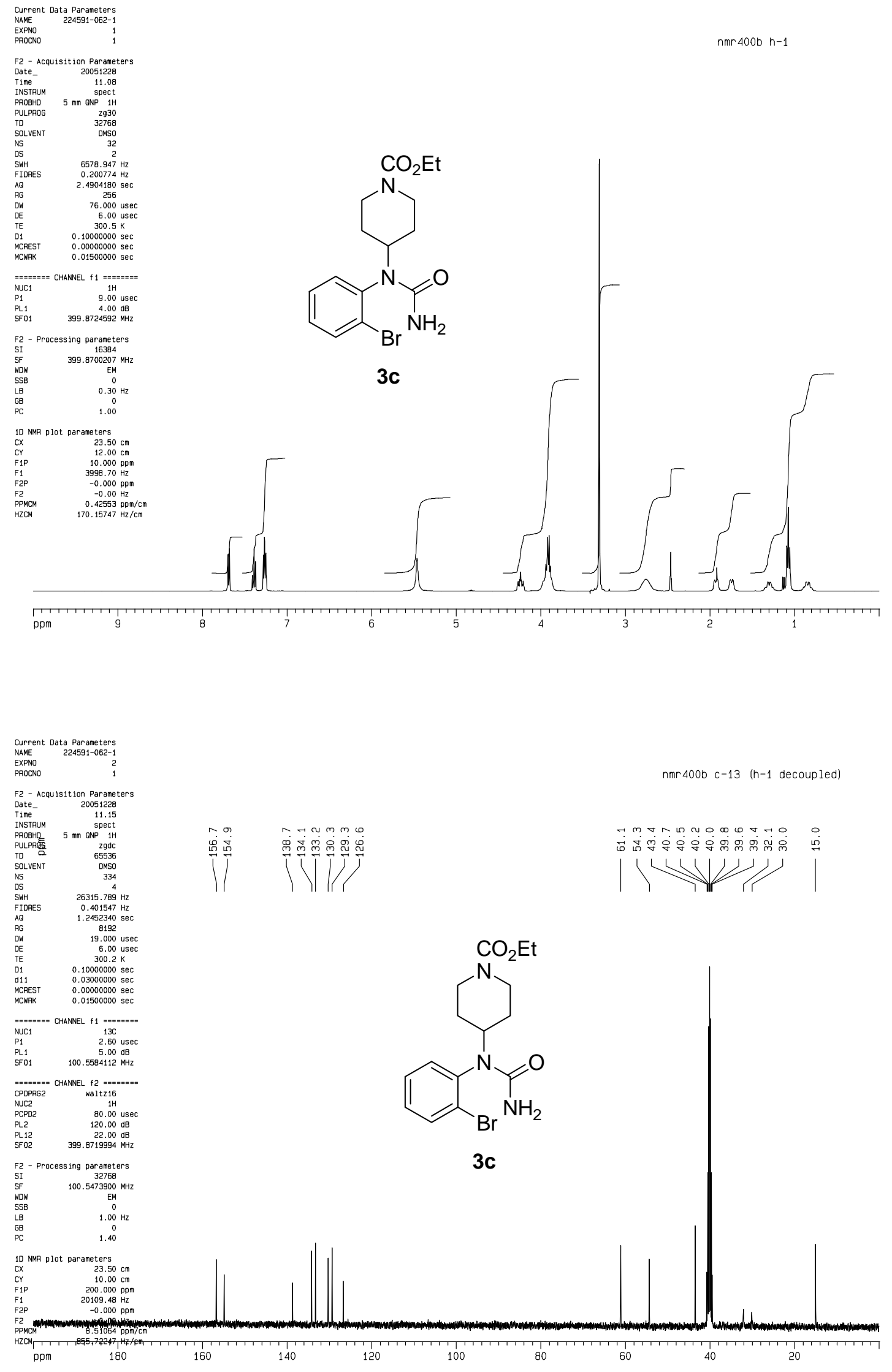



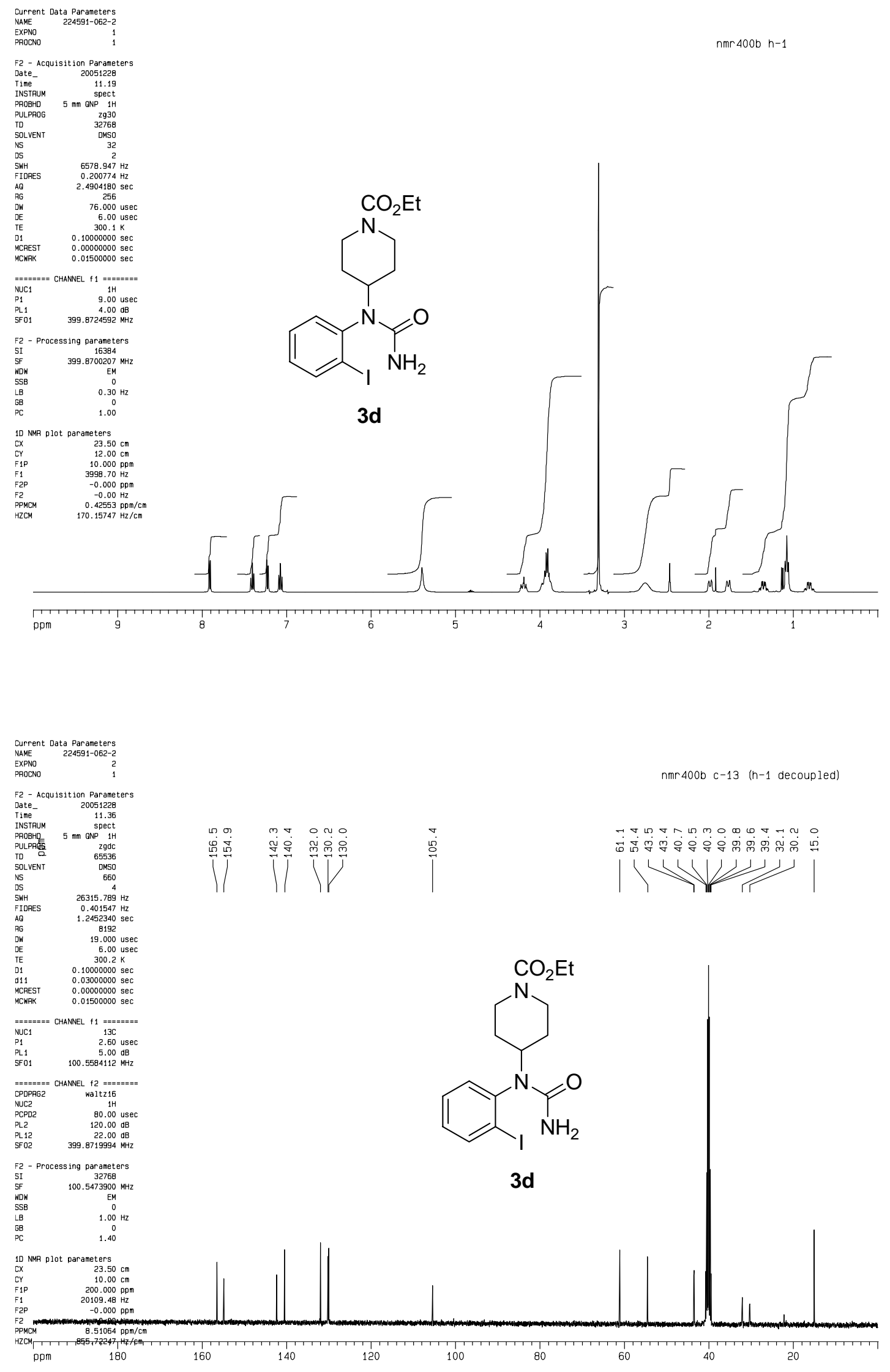

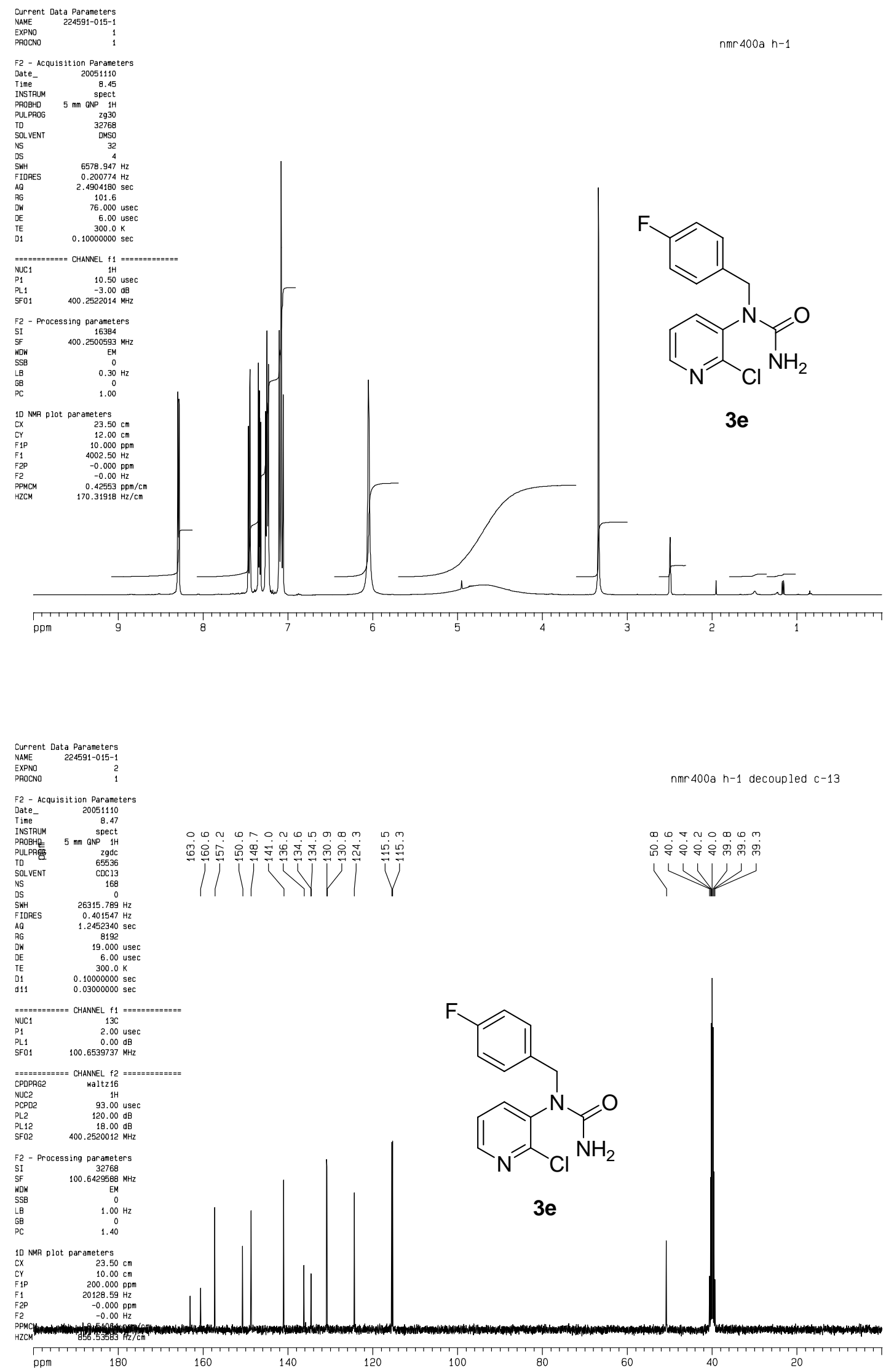

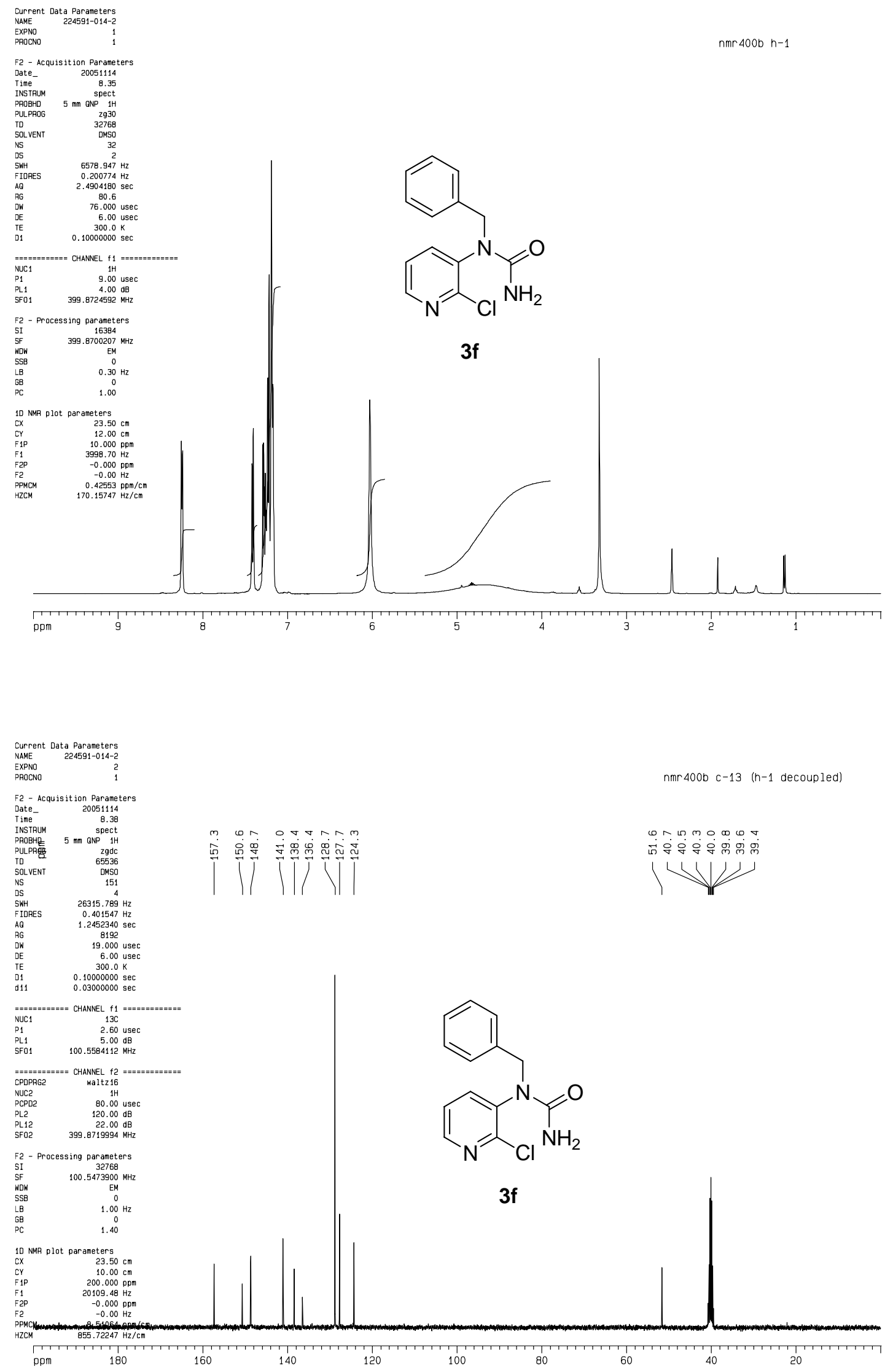

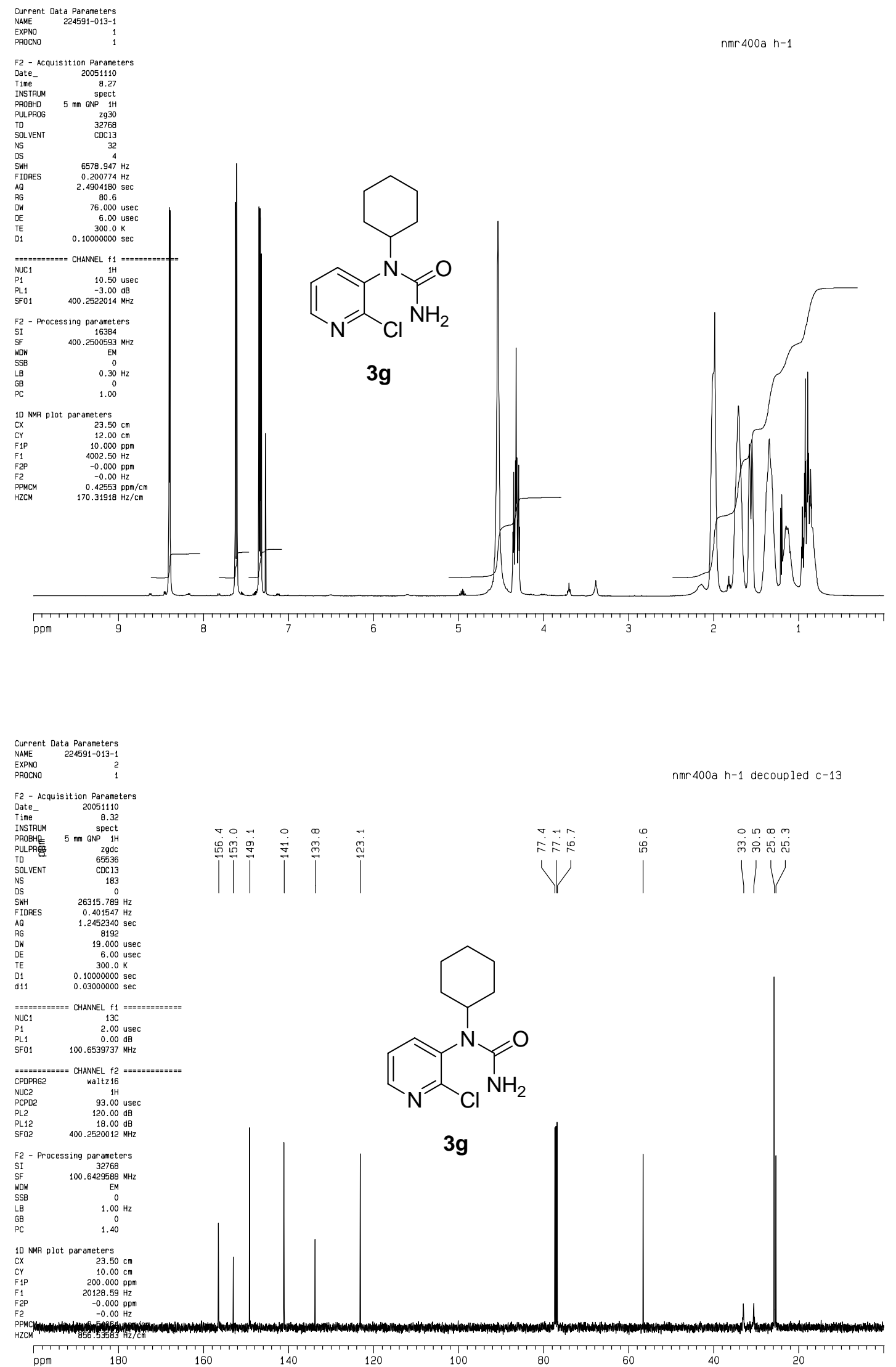

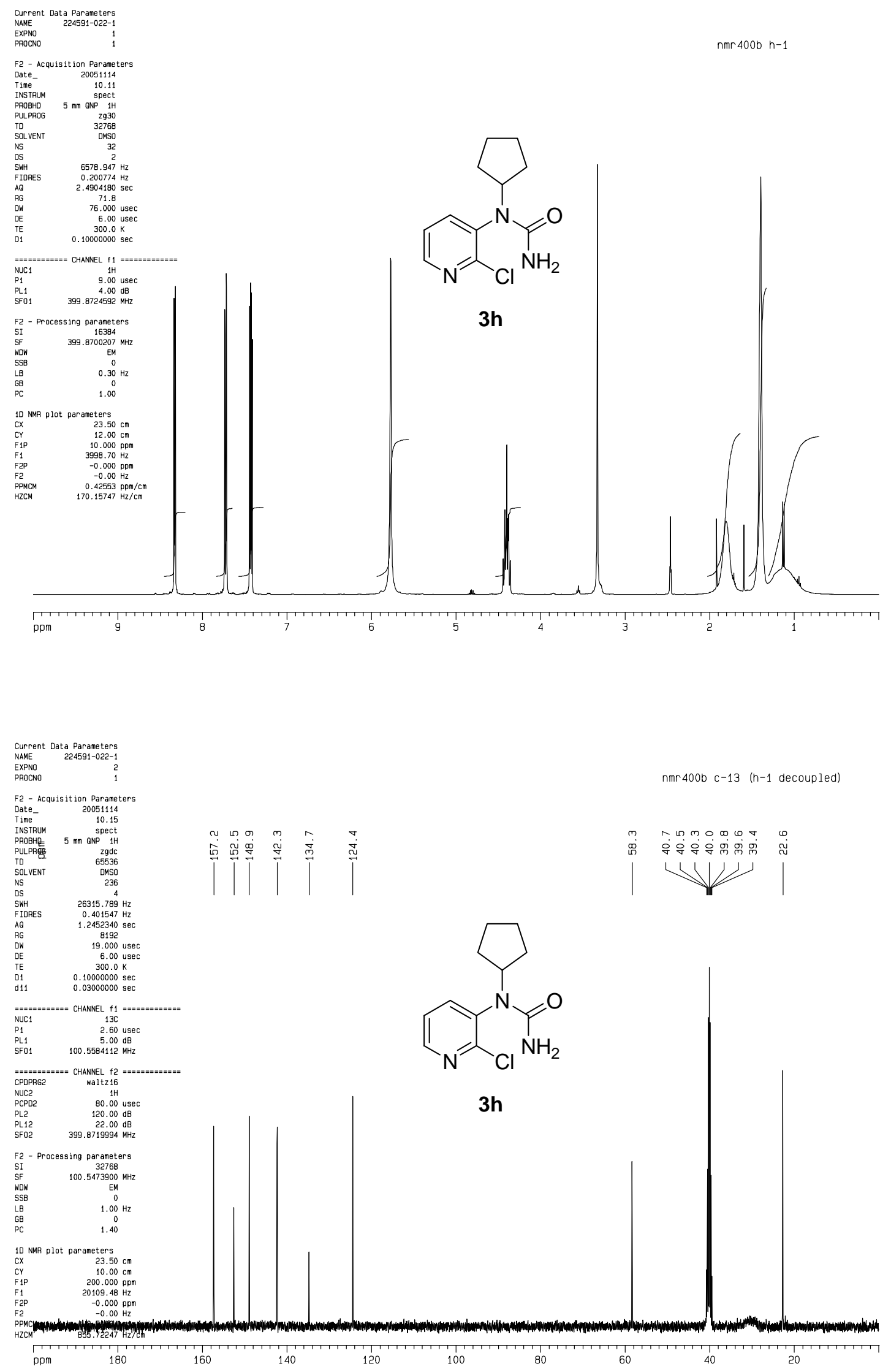

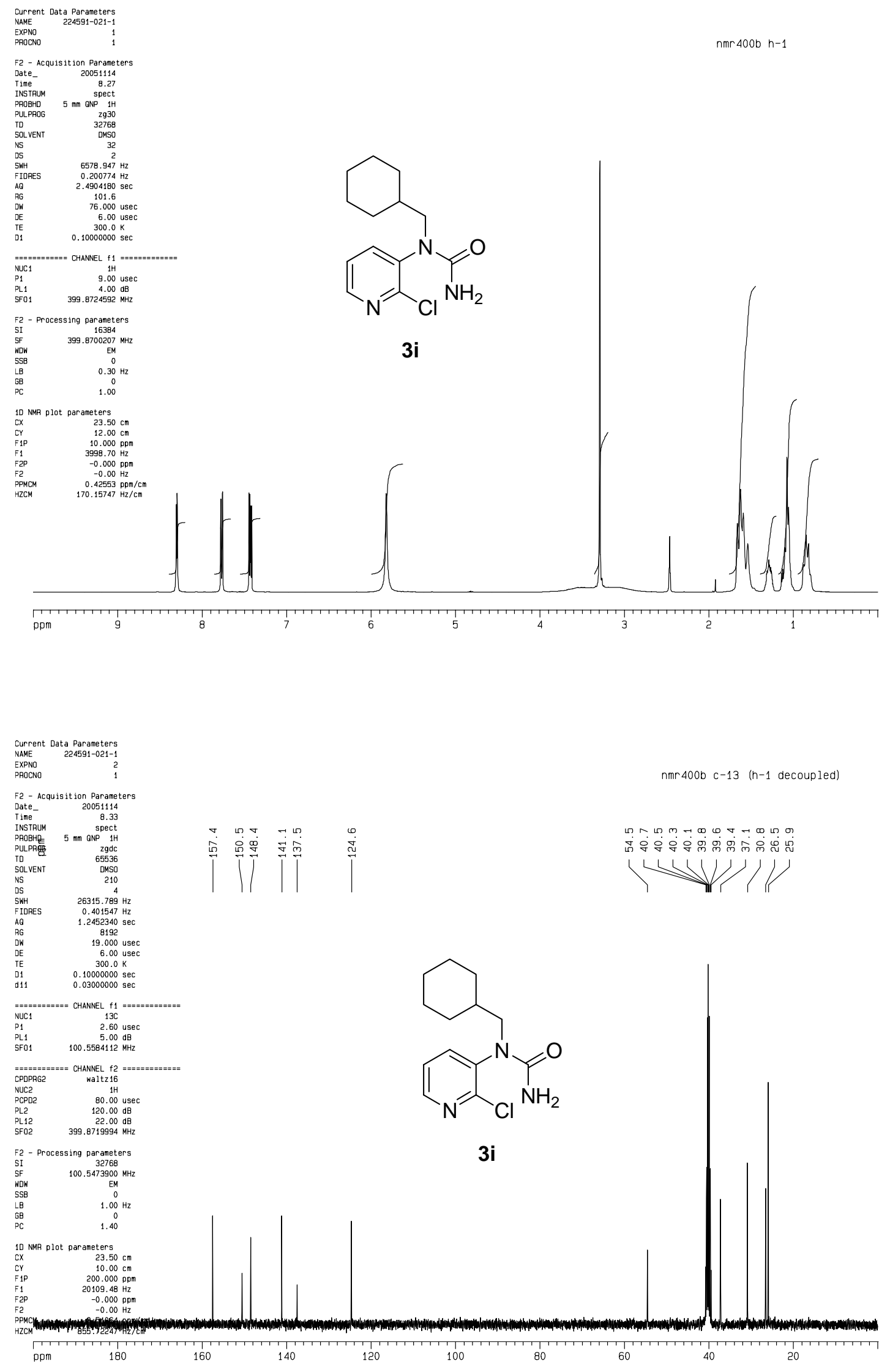

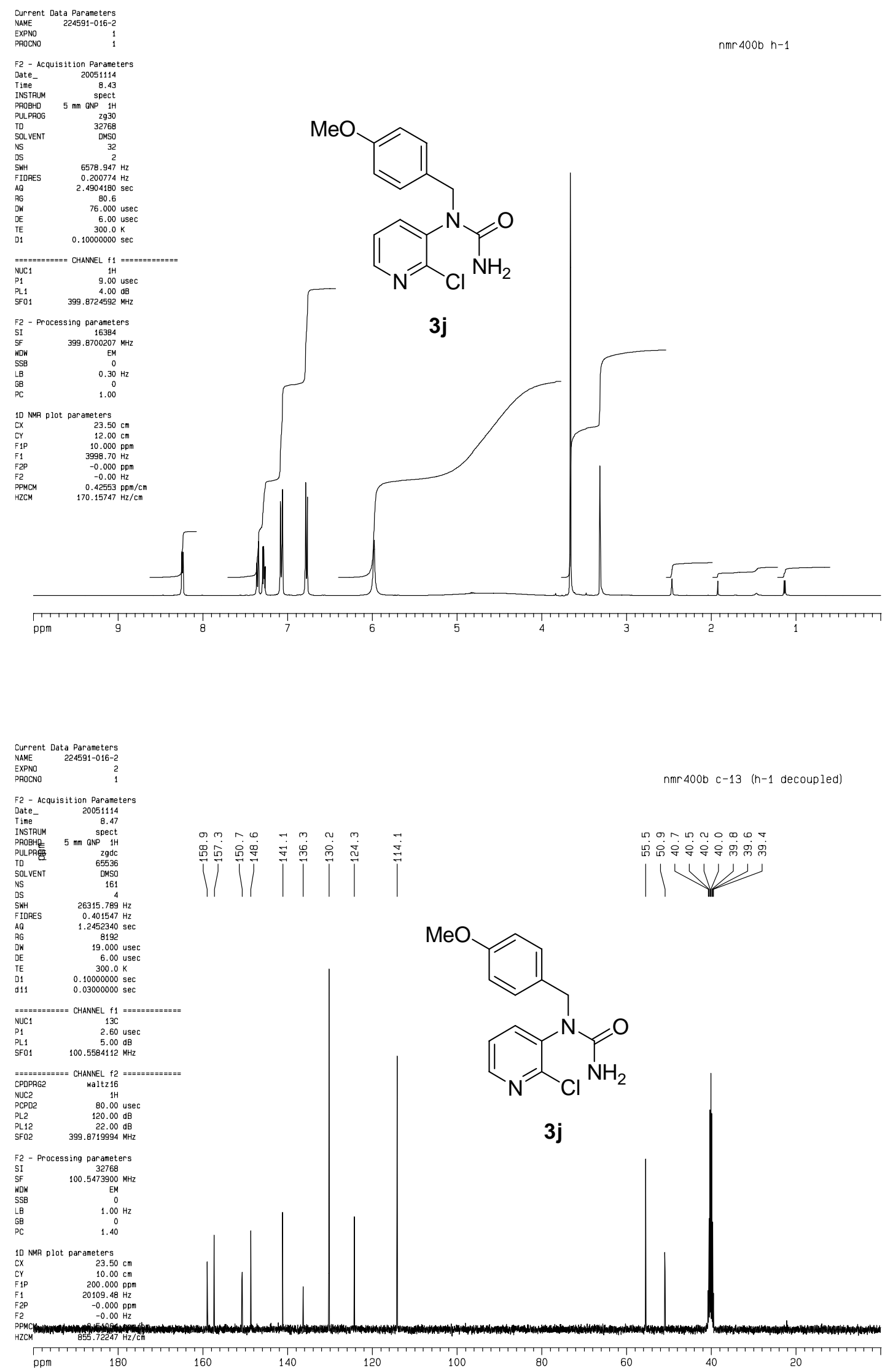

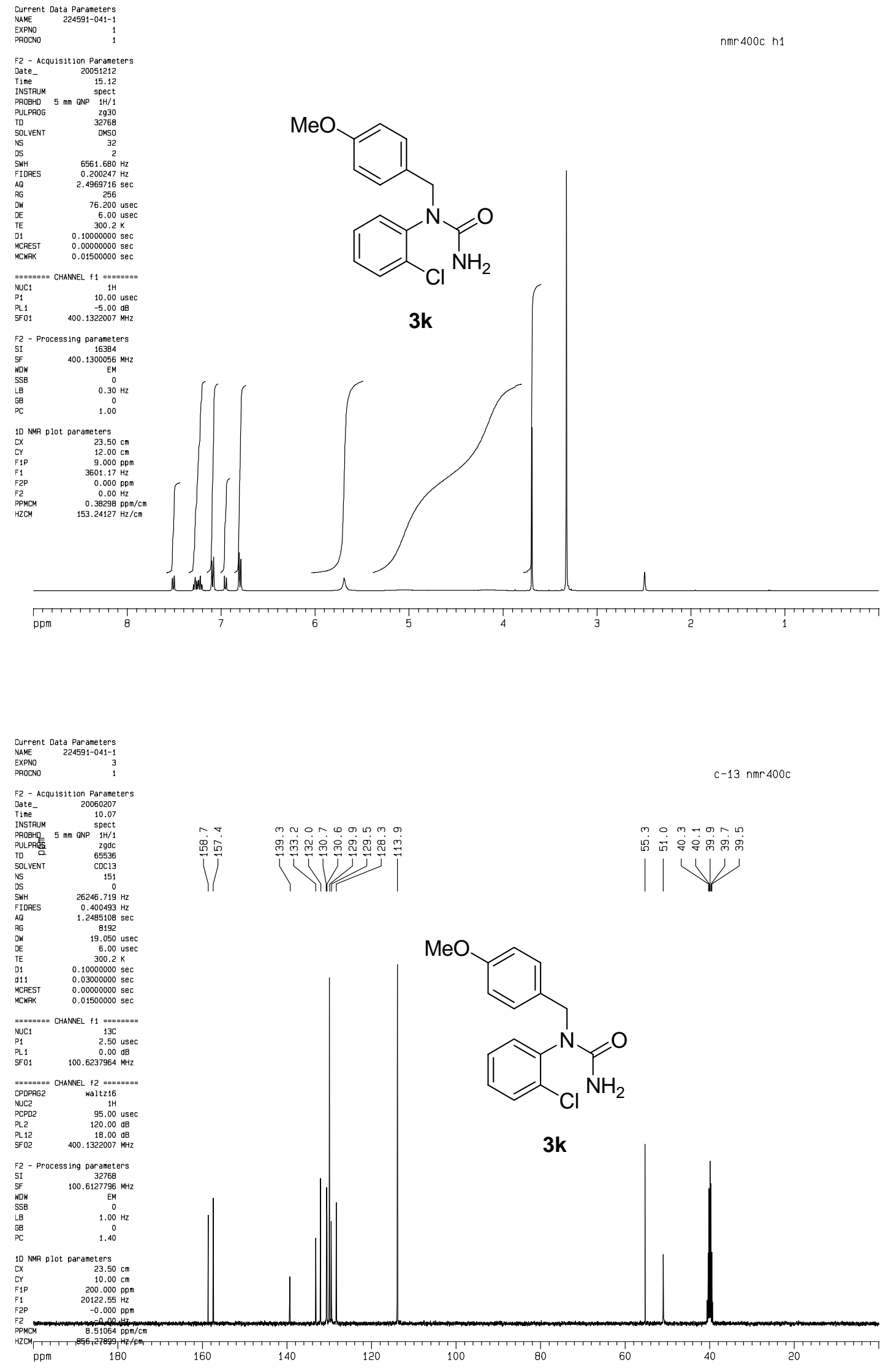

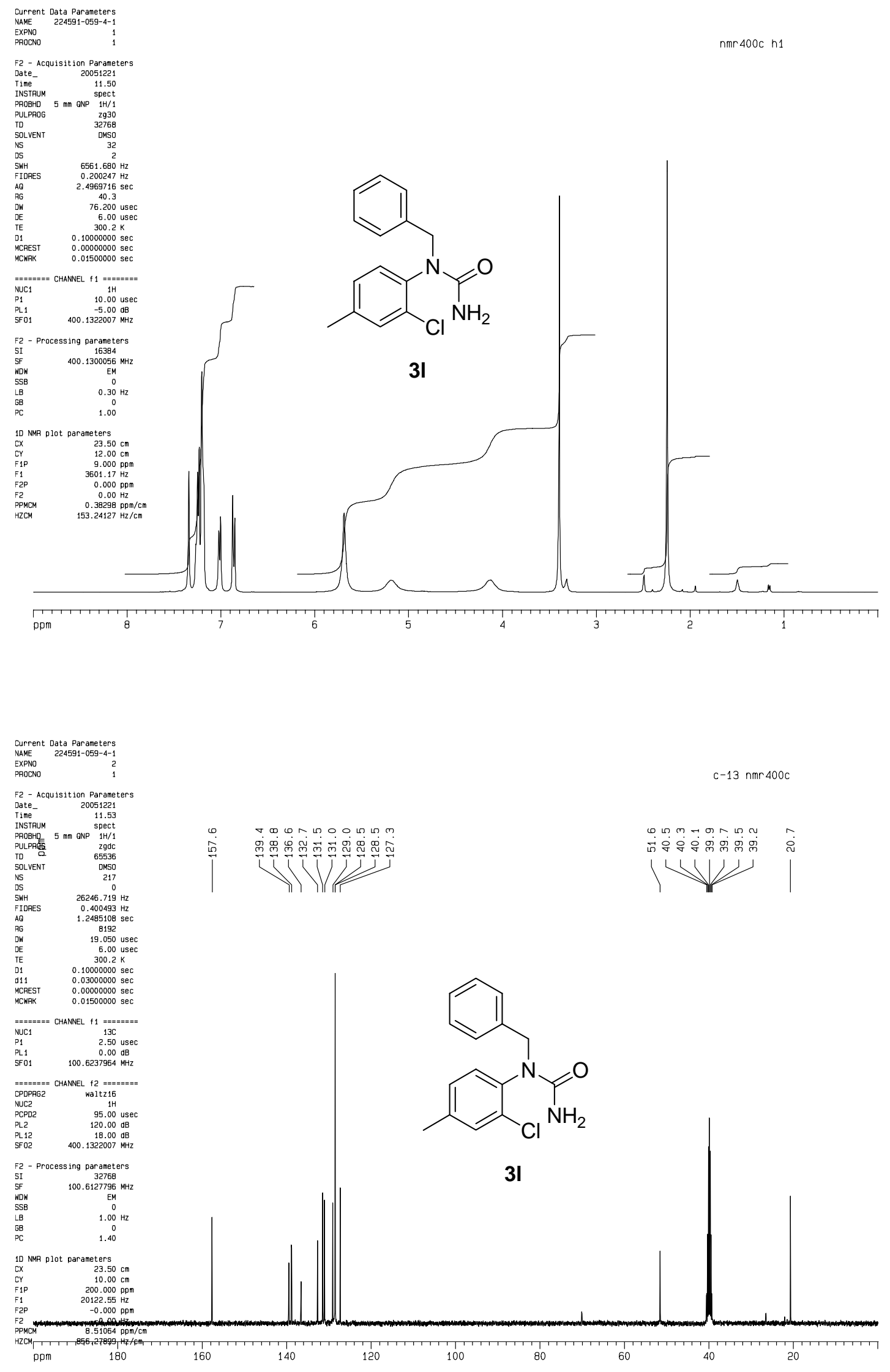

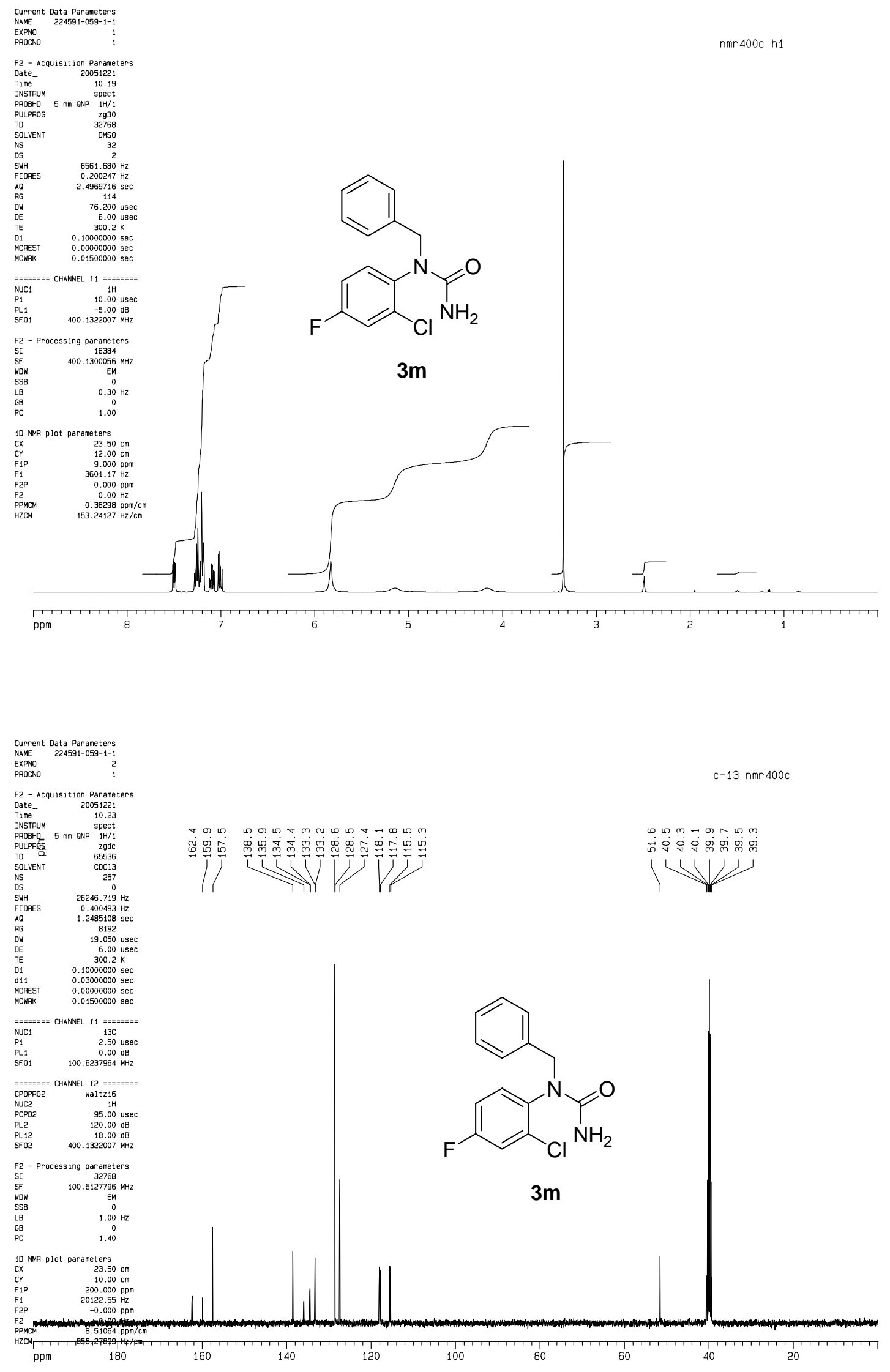

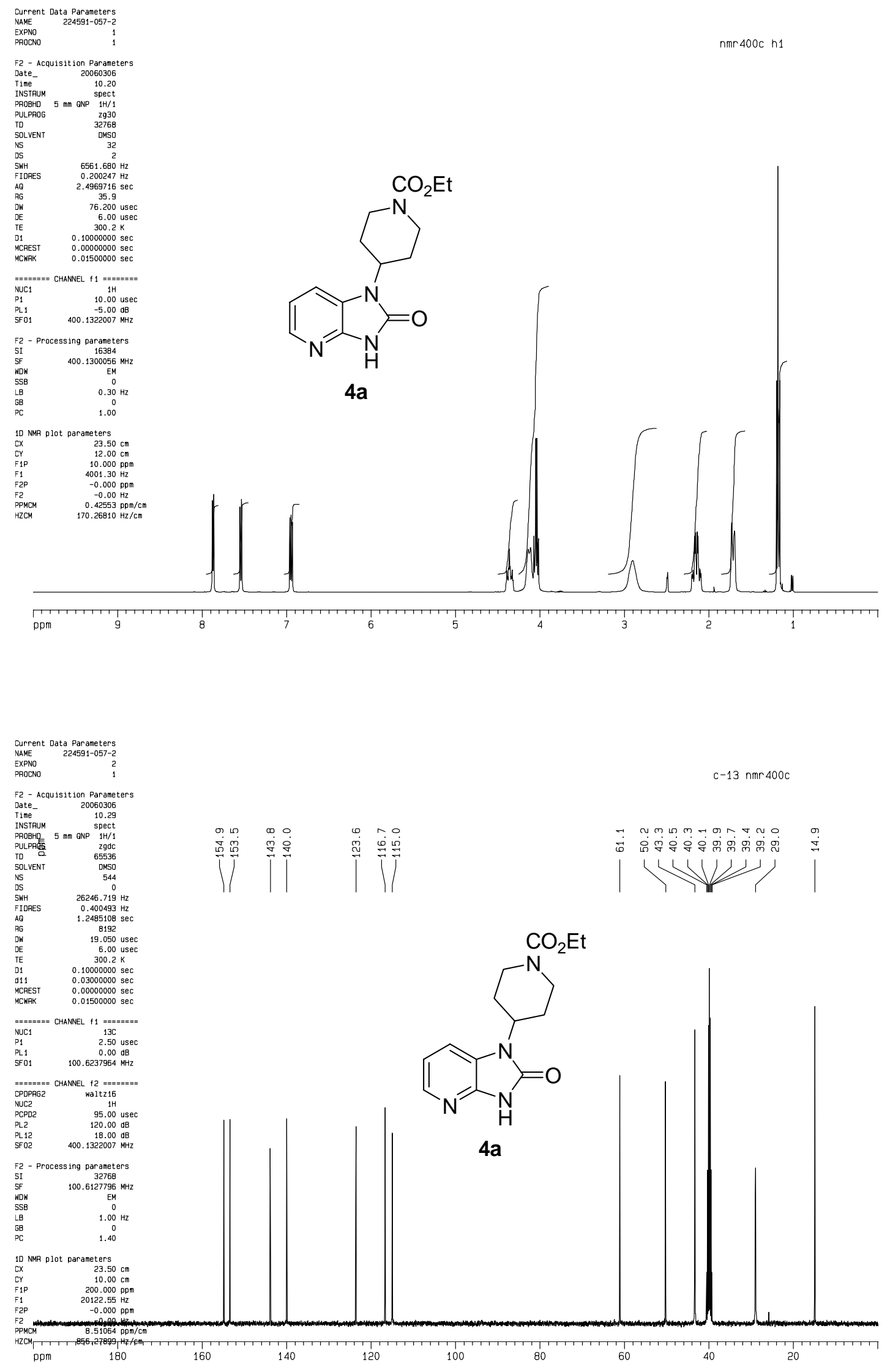

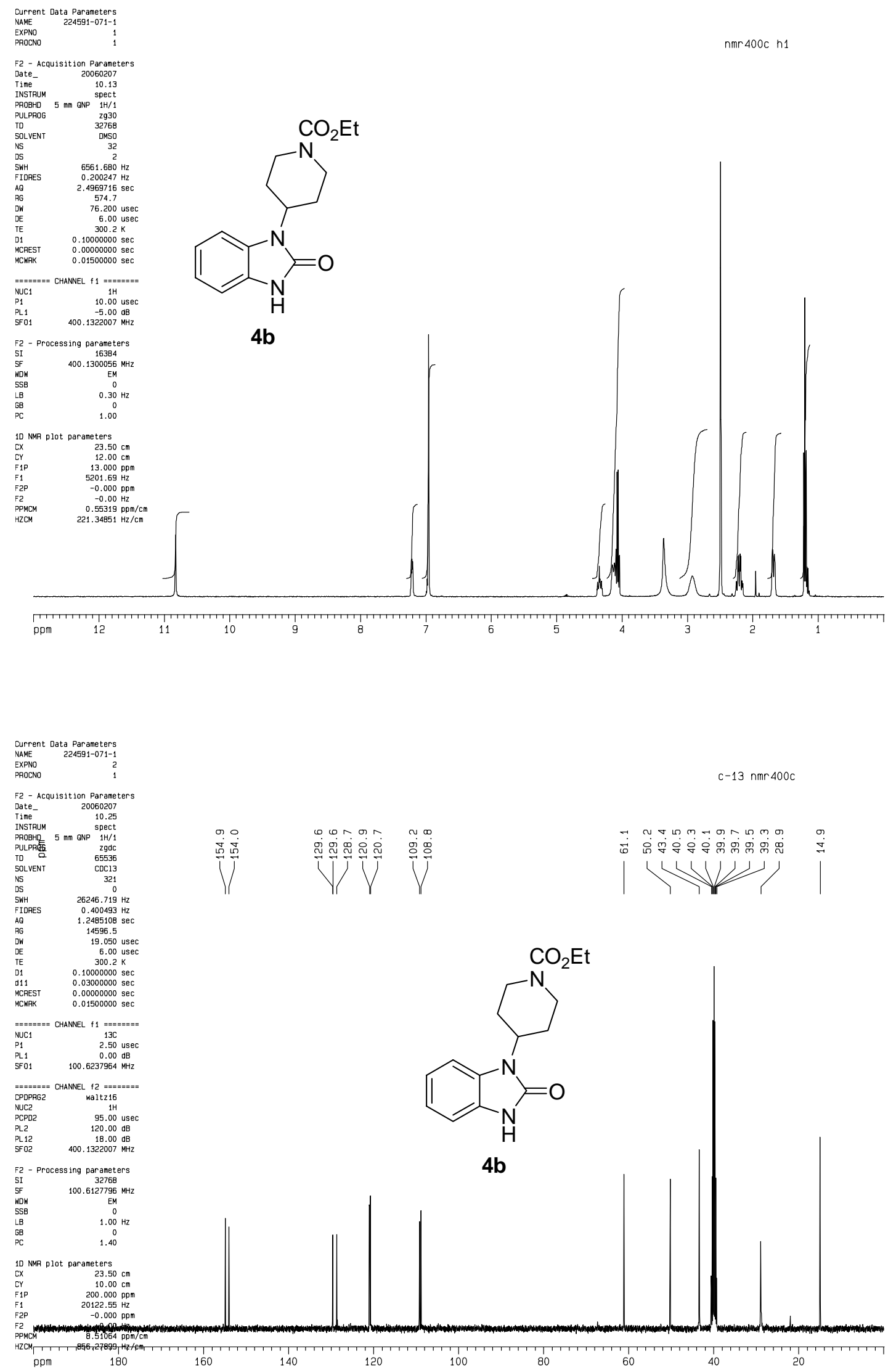

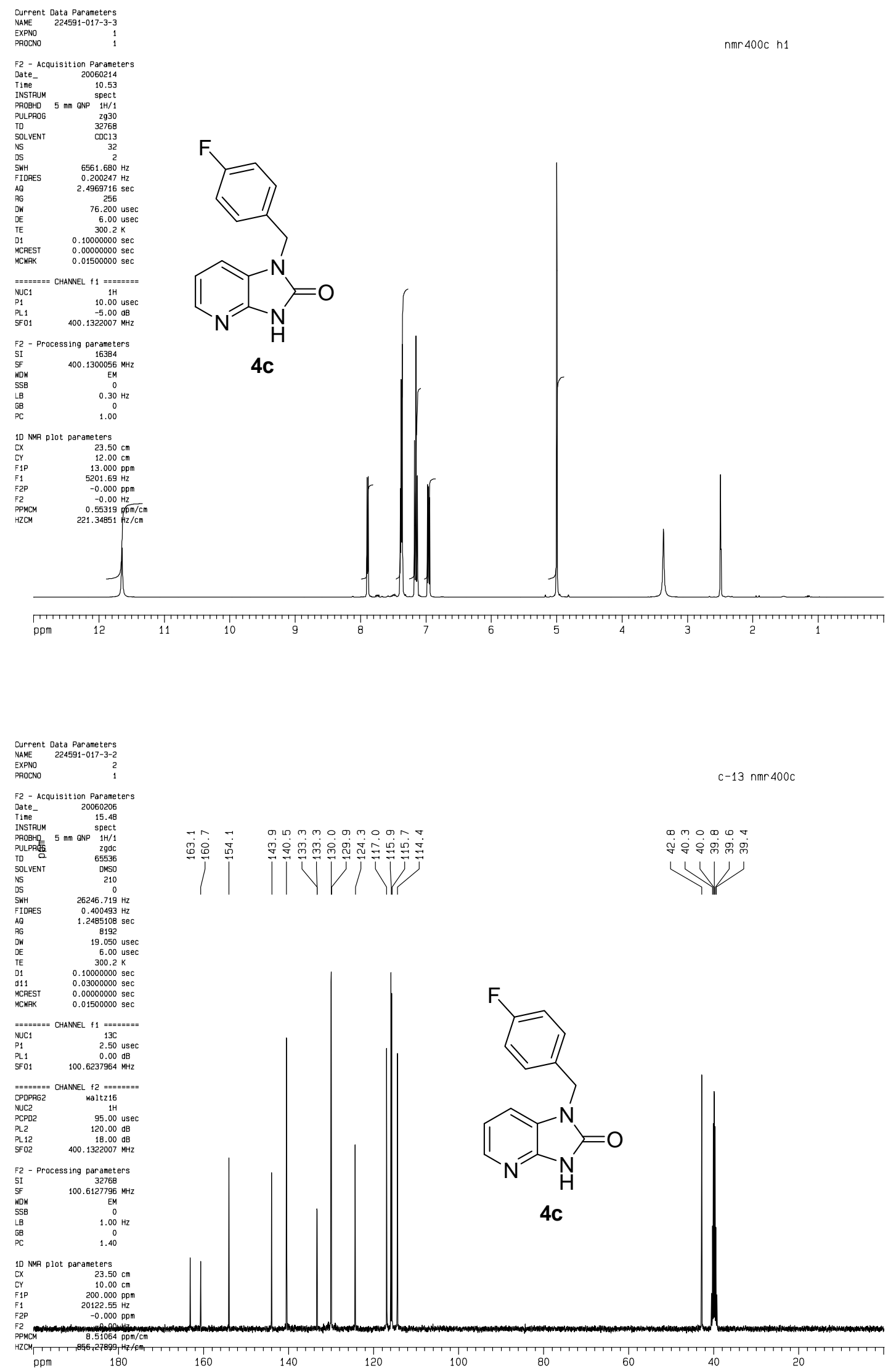

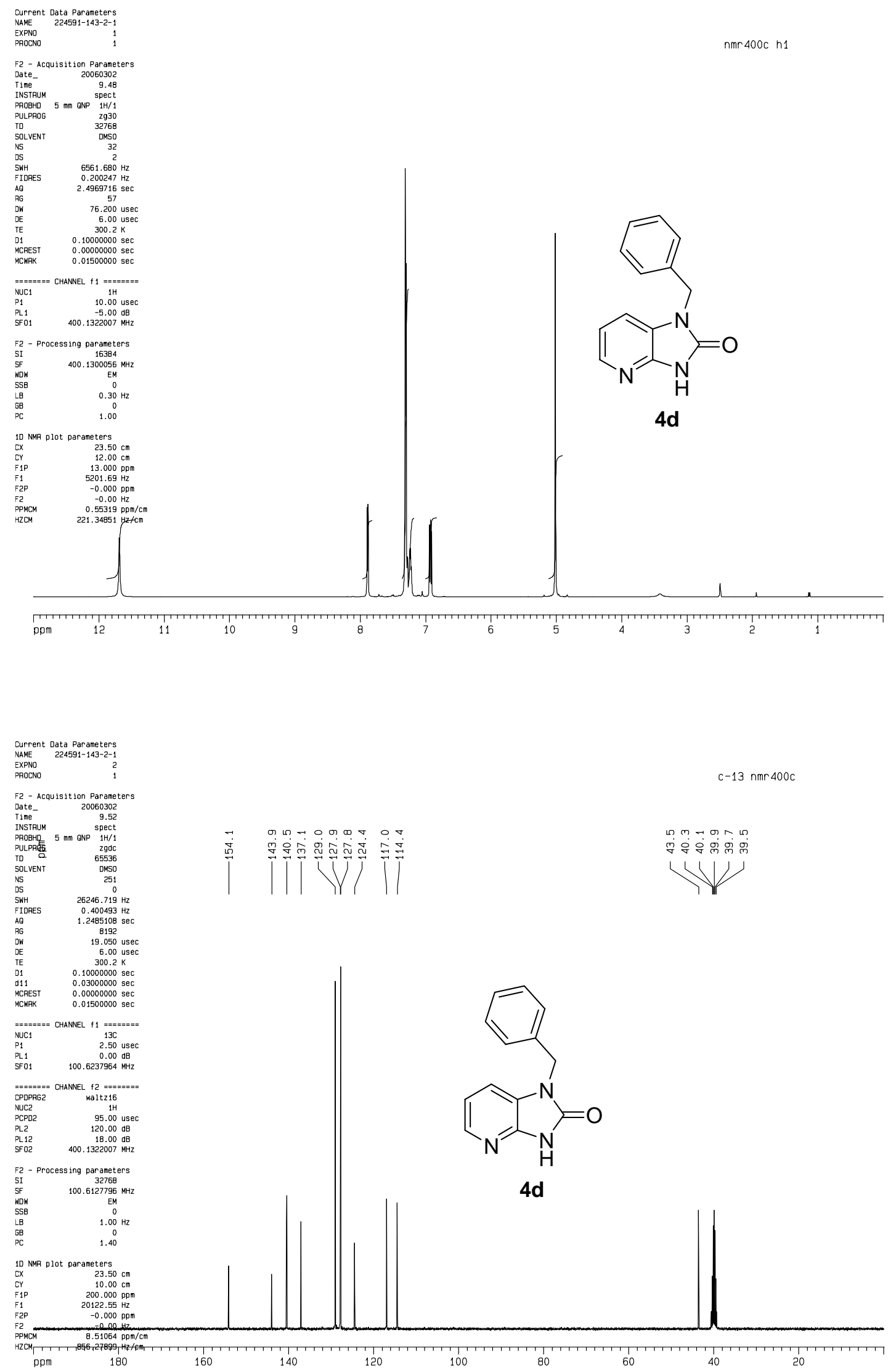

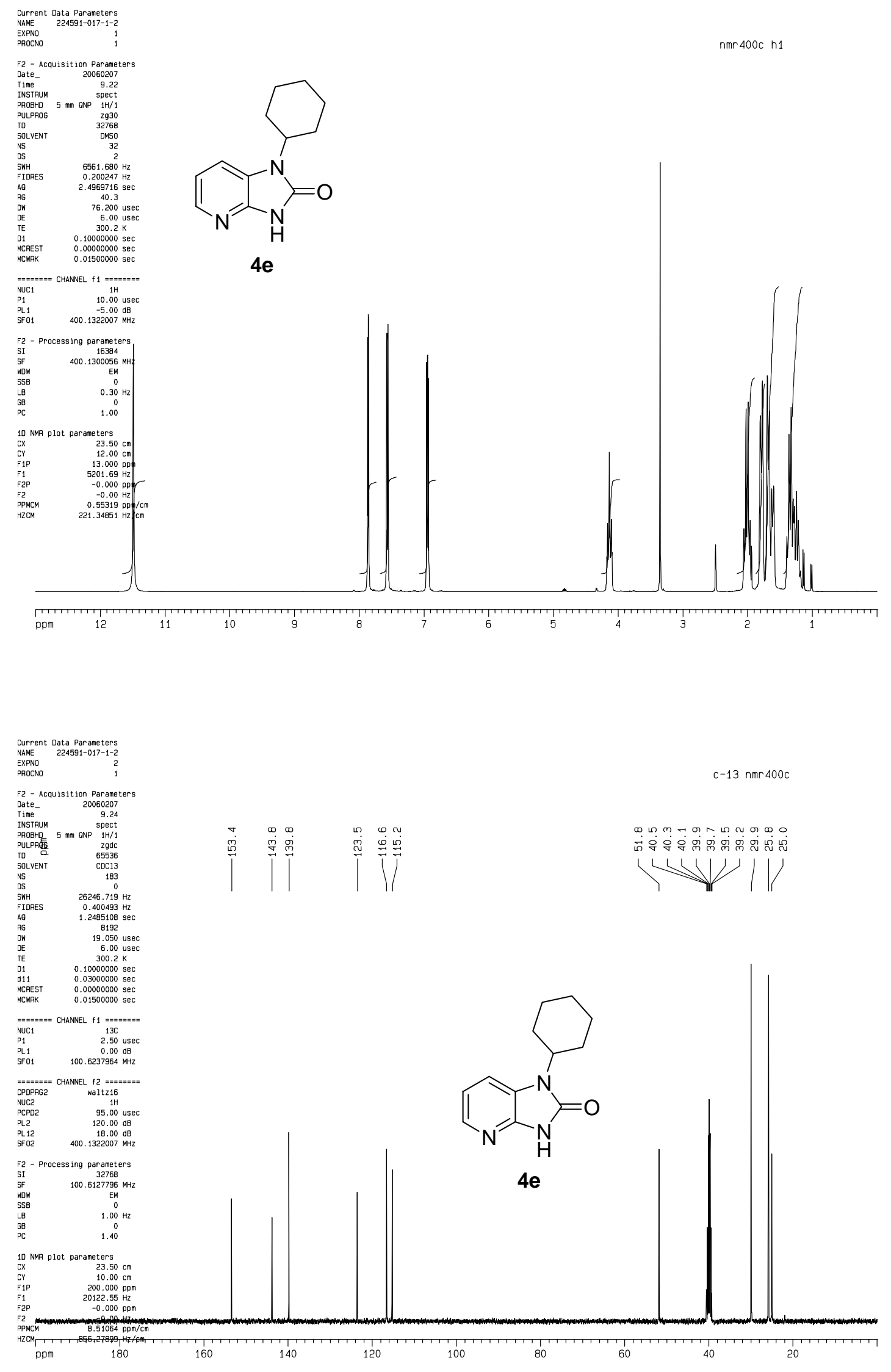

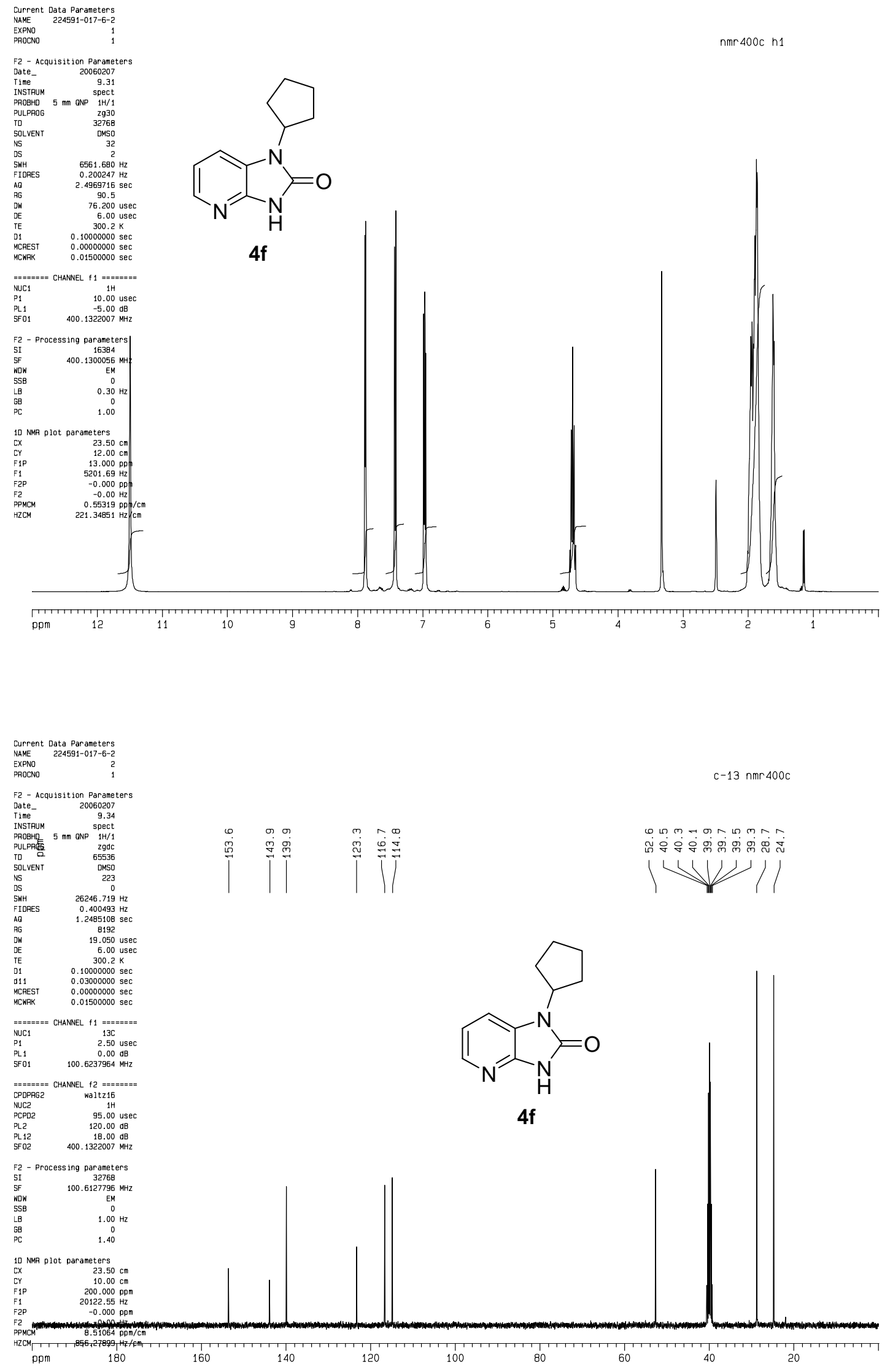

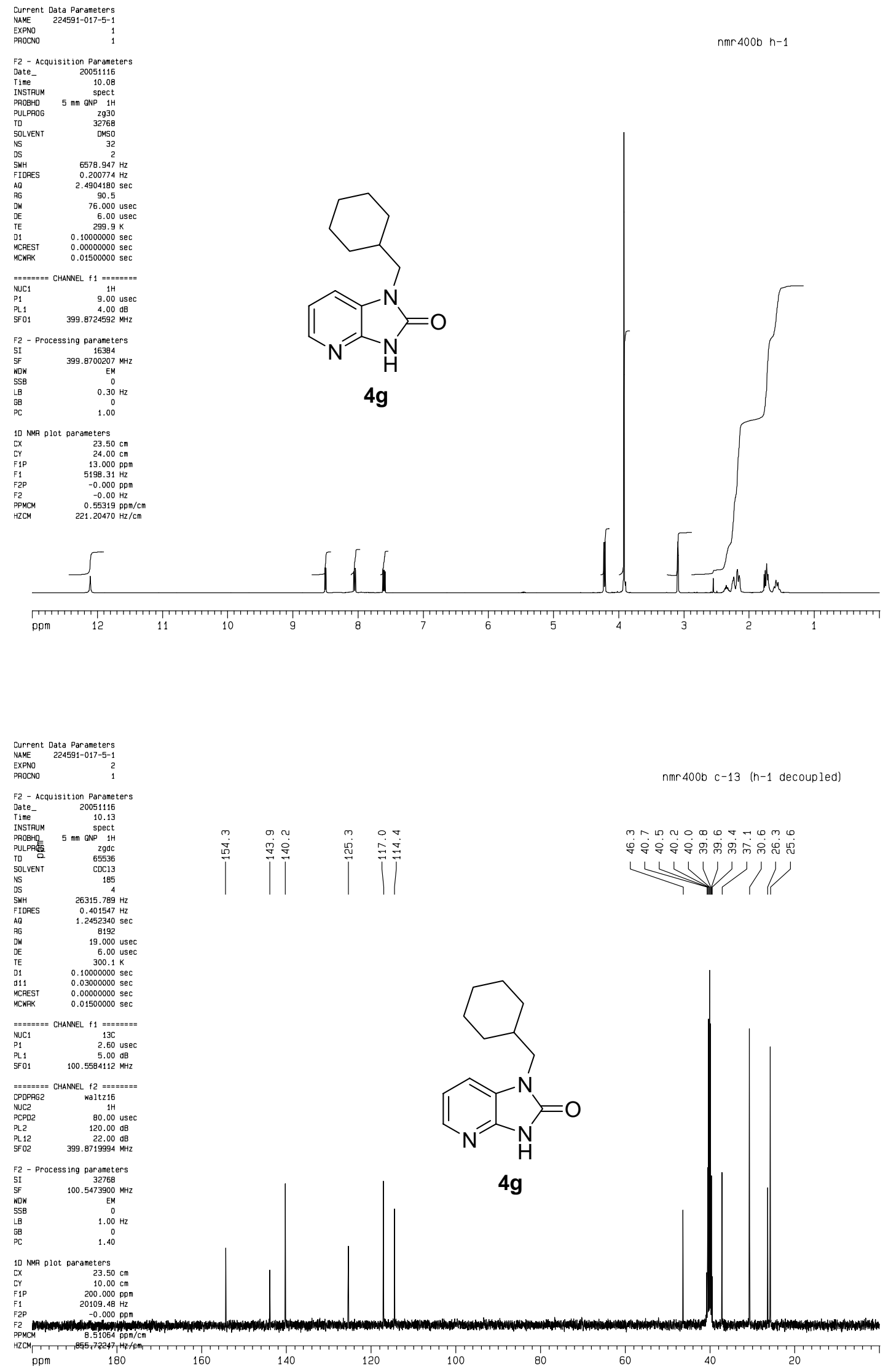

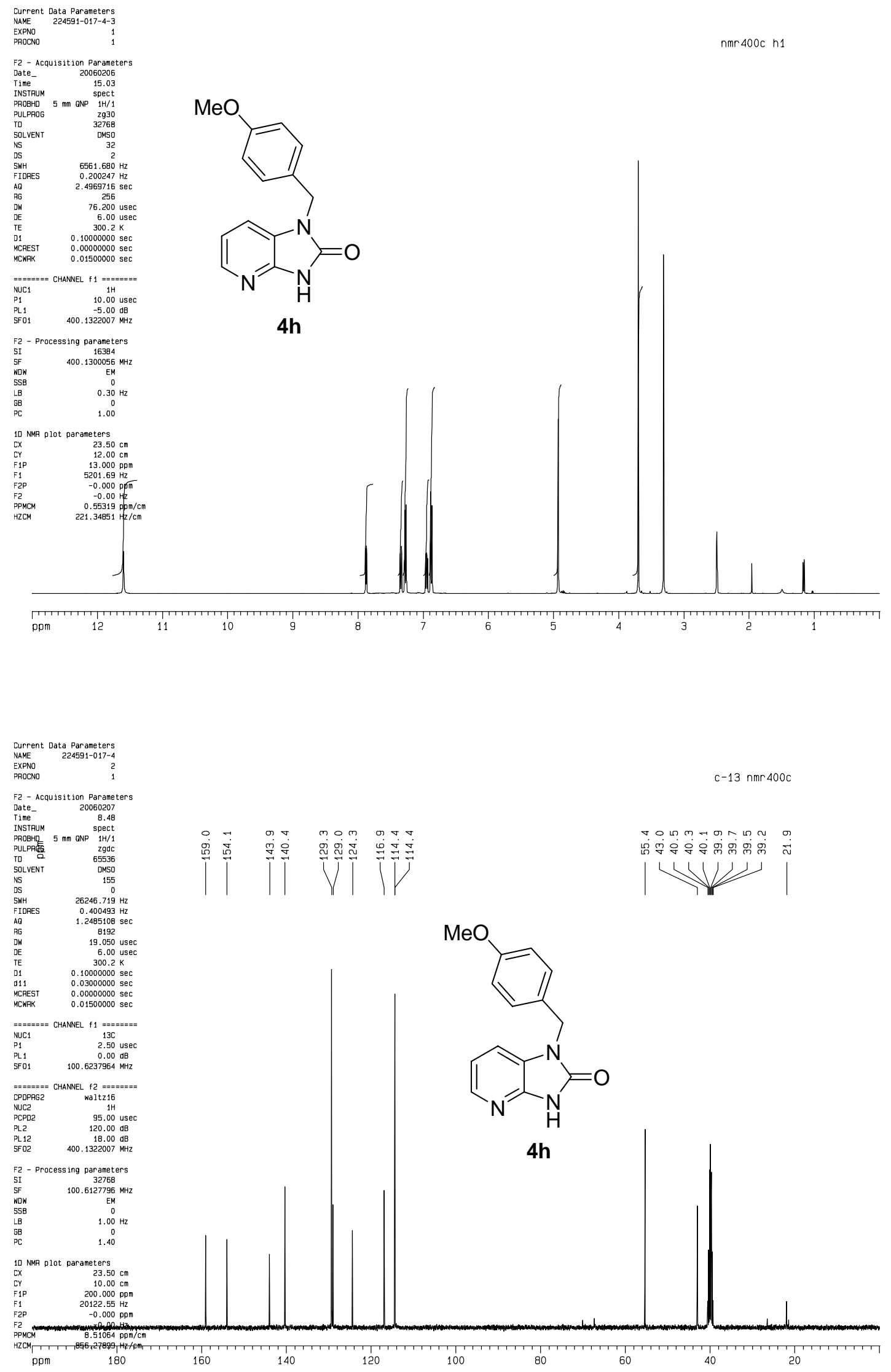

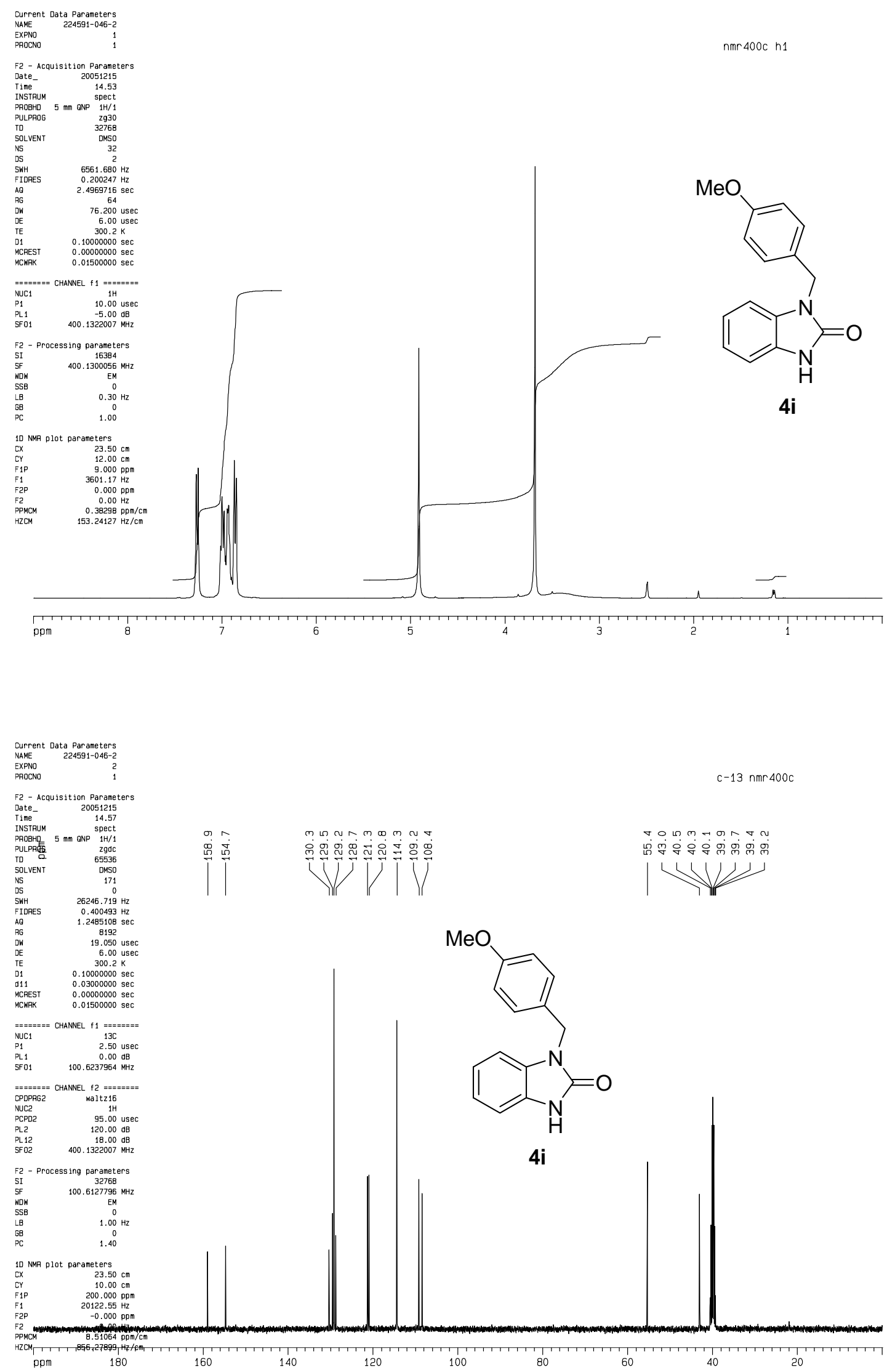

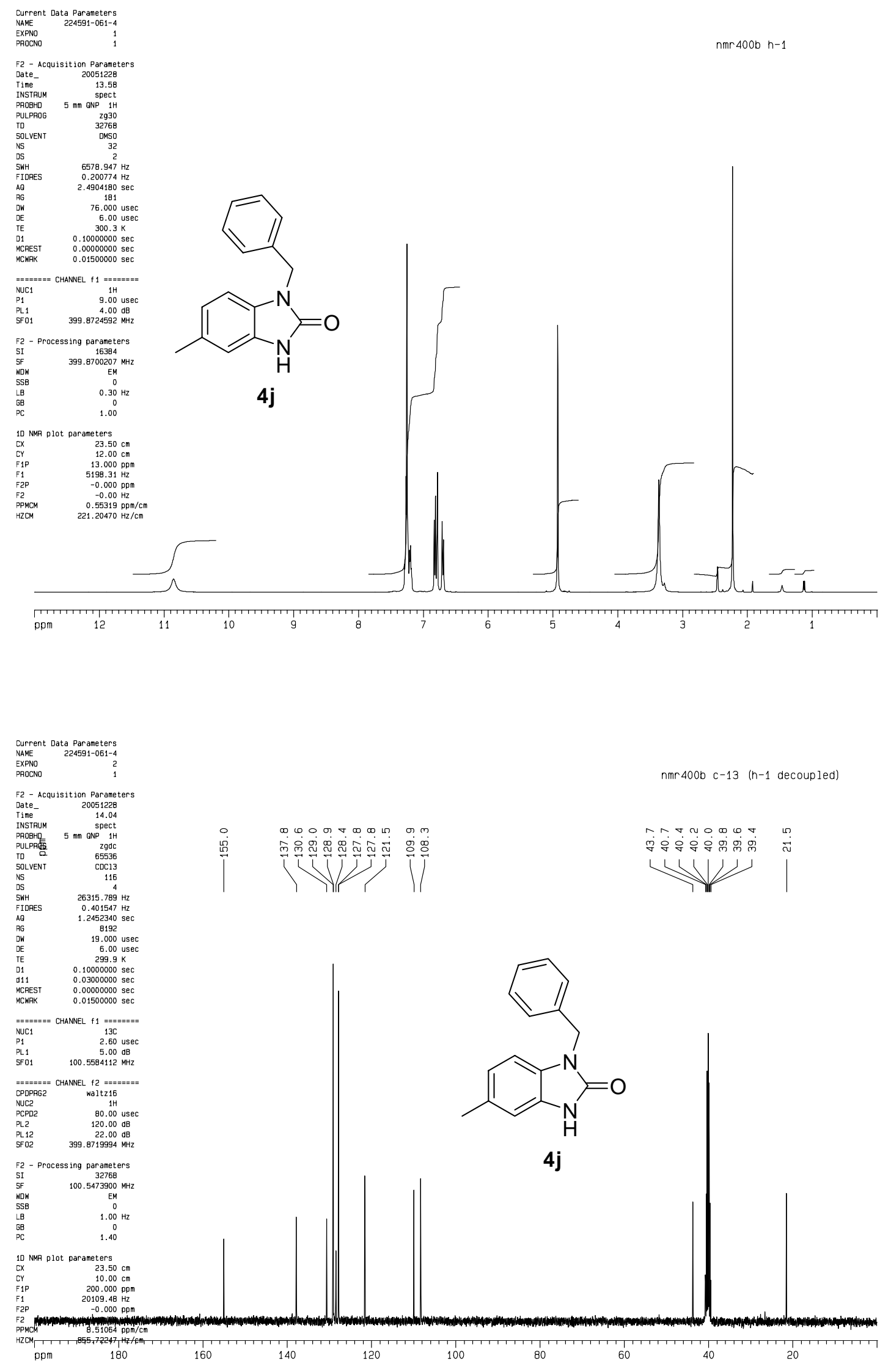

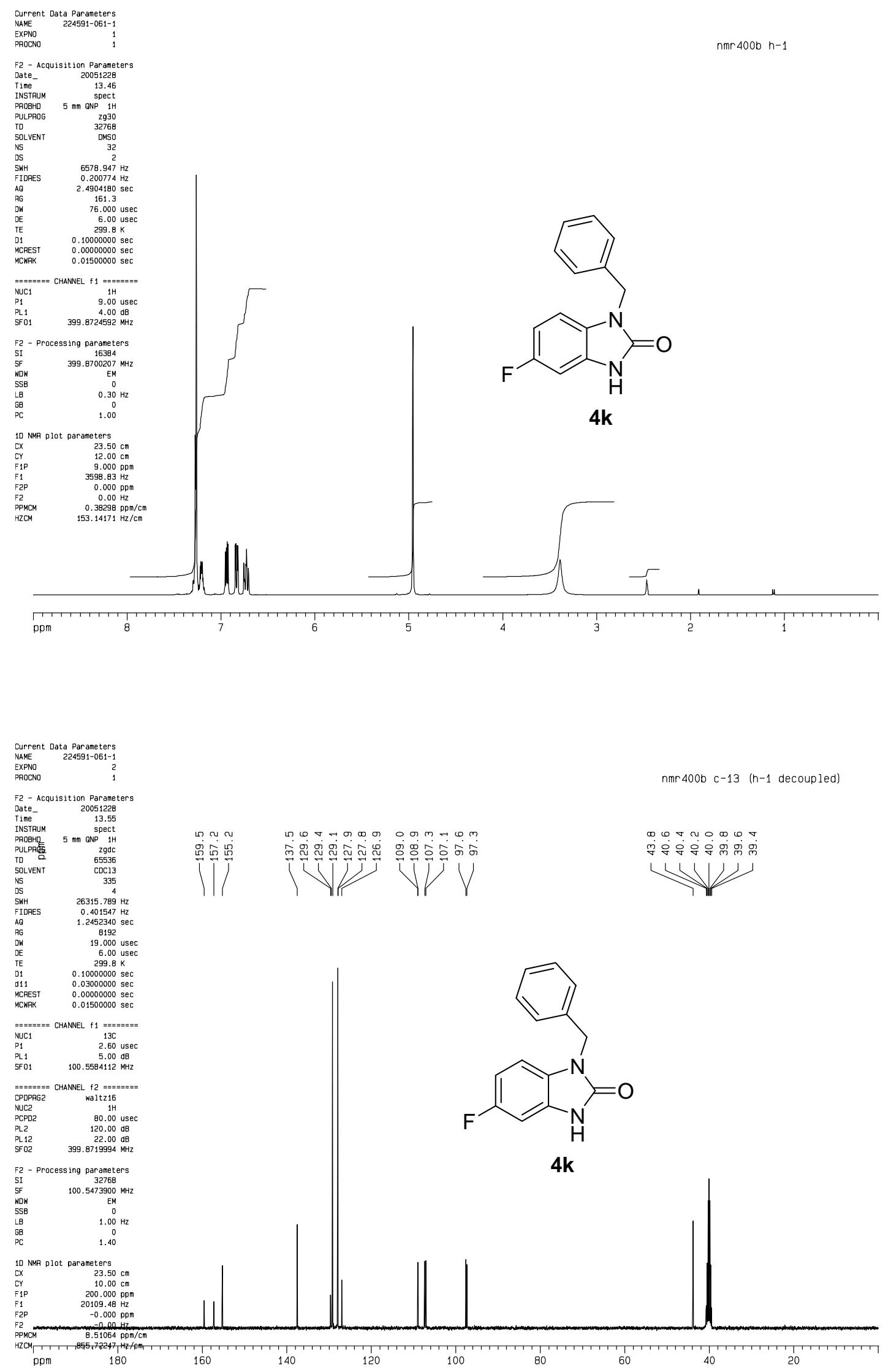